Article

\title{
Anomaly-Induced Transport Phenomena from Imaginary-Time Formalism
}

\author{
Masaru Hongo $1,2, *\left(\mathbb{D}\right.$ and Yoshimasa Hidaka ${ }^{1,3}$ \\ 1 RIKEN iTHEMS, RIKEN, Wako, Saitama 351-0198, Japan; hidaka@riken.jp \\ 2 Research and Education Center for Natural Sciences, Keio University, Yokohama, Kanagawa 223-8521, Japan \\ 3 Quantum Hadron Physics Laboratory, RIKEN Nishina Center, RIKEN, Wako, Saitama 351-0198, Japan \\ * Correspondence: masaru.hongo@riken.jp; Tel.: +81-48-462-1226
}

Received: 25 February 2019; Accepted: 6 May 2019; Published: 16 May 2019

check for updates

\begin{abstract}
A derivation of anomaly-induced transport phenomena-the chiral magnetic/vortical effect—is revisited based on the imaginary-time formalism of quantum field theory. Considering the simplest anomalous system composed of a single Weyl fermion, we provide two derivations: perturbative (one-loop) evaluation of the anomalous transport coefficient, and the anomaly matching for the local thermodynamic functional.
\end{abstract}

Keywords: finite temperature field theory; path integrals; quantum fields in curved spacetime; quantum statistical mechanics; symmetries; quantum anomalies; hydrodynamics

\section{Introduction}

Quantum anomaly is one of the most fundamental properties of quantum systems, which keeps staying in the low-energy regime once it appears in an underlying UV theory [1,2]. As a consequence, the low-energy dynamics is strongly influenced by the existence of the quantum anomaly. A well-known example is the chiral anomaly in QCD, which gives rise to the Wess-Zumino term in the low-energy effective theory of QCD (the chiral perturbation theory) describing the neutral pion decay into two photons $\left(\pi^{0} \rightarrow \gamma \gamma\right)$ [3-5]. The notion of anomaly can be generalized to discrete symmetries of systems such as time-reversal symmetry. The anomaly matching argument [6,7] is actively applied to restrict the possible nontrivial ground states (See Refs. [8-19] for recent applications).

It has been recently noticed that quantum anomaly also appears even in the effective theory describing the real-time dynamics of nonequilibrium systems, e.g., hydrodynamics and the kinetic theory, and it affects the macroscopic transport properties in the hydrodynamic regime [20-61] (See also pioneering works by Vilenkin [62,63]). For example, the simplest anomalous system composed of a single right-handed Weyl fermion coupled to a background electromagnetic field shows interesting transport. When this system is put into an environment with a temperature $T$ and a chemical potential $\mu_{R}$, the chiral anomaly induces the dissipationless current along the magnetic field $B^{i}$ given by

$$
\left\langle\hat{J}_{R}^{i}\right\rangle_{\text {ano }}=\sigma_{B} B^{i}+\sigma_{\omega} \omega^{i} \quad \text { with } \quad \sigma_{B}=\frac{\mu_{R}}{4 \pi^{2}}, \quad \sigma_{\omega}=\frac{\mu_{R}^{2}}{4 \pi^{2}}+\frac{T^{2}}{12},
$$

where $\left\langle\hat{J}_{R}^{\mu}\right\rangle_{\text {ano }}$ denotes the anomalous part of the expectation value of the right-handed current, and $\sigma_{B}$ $\left(\sigma_{\omega}\right)$ is regarded as the chiral magnetic (vortical) conductivity. The first and second terms in Equation (1) are called the chiral magnetic effect (CME) and chiral vortical effect (CVE), respectively (See Figure 1). It is worth pointing out that even in the weak coupling limit, $\sigma_{B}$ and $\sigma_{\omega}$ do not diverge unlike the usual conductivity because their existence is protected by the quantum anomaly.

These anomalous transports are believed to be universally present when the system under consideration contains the chiral anomaly. For example, they are expected to take place in the 
quark-gluon plasma created in high-energy heavy-ion collisions [64-74], astrophysical plasma including neutrino process [75-80], and Weyl semimetals realized in condensed matter physics [81-90]. While we have not observed clear experimental signal of the anomaly-induced transport in the first two systems, it has been recently reported that the experimental signals of the CME are achieved in the Weyl semimetal [91-93].

(a) Chiral Magnetic Effect

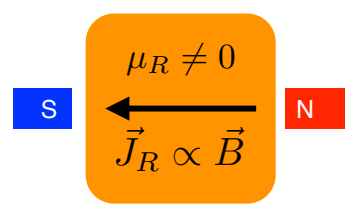

(b) Chiral Vortical Effect

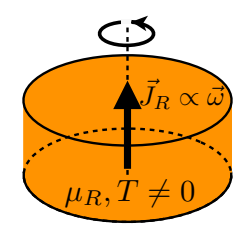

Figure 1. The schematic picture of the anomaly-induced transport phenomena: (a) Chiral magnetic effect. (b) Chiral vortical effect.

The theoretical derivation of the anomaly-induced transport phenomena has been remarkably developed in the past ten years, e.g., the direct field theoretical evaluation [20], the fluid/gravity correspondence [21-23,25], the phenomenological entropy-current analysis [24], the linear response theory $[26,31,34,50,58]$, the kinetic theory $[27,28,33,36,37,41,42,44,48,49,51-53,55,57,59-61]$, and the hydrostatic partition function method and extensions [29,30,32,35,38-40,43,45,46,54]. In this paper, we review the derivation of the anomaly-induced transport phenomena from the statistical mechanical viewpoint with the help of the imaginary-time (Matsubara) formalism of quantum field theory [94-97]. In particular, we demonstrate two derivations, which are basically on the same line as the last two derivations raised above. For that purpose, we consider the simplest anomalous system composed of a single Weyl fermion coupled to an external electromagnetic field. Although most results given in this paper has been already known, we give the clear rigorous justification of the hydrostatic partition function method for the anomalous system based on the statistical ensemble describing systems in general local thermal equilibrium. This shows that the hydrostatic partition function method is indeed not restricted to the real hydrostatic situation, but applicable to systems in general local thermal equilibrium.

The paper is organized as follows: In Section 2, we review the basic setup and formulation including the Zubarev's nonequilibrium statistical operator methods [98-100] (See also Refs. [101-104] for a recent sophisticated revival of a similar idea). In Section 3, we then provide the perturbative evaluation of the chiral magnetic/vortical conductivity with the help of the (equilibrium) linear response theory, from which we can read off the constitutive relation for the anomalous current. In Section 4, we give another nonperturbative derivation based on the anomaly matching for the local thermodynamic functional. Section 5 is devoted to the summary and discussion.

\section{Preliminaries for the Anomaly-Induced Transport Phenomena}

In this section, we briefly summarize the formulation to derive the anomaly-induced transport phenomena based on the imaginary-time formalism [98-104].

\subsection{Anomalous (Non-)Conservation Laws for a Single Weyl Fermion}

Let us consider the system consisting of a right-handed Weyl fermions $\xi$ under an external $U(1)$ gauge field $A_{\mu}$ in a $(3+1)$ dimensional curved spacetime, whose action has the form:

$$
\mathcal{S}\left[\xi, \xi^{\dagger} ; A_{\mu}, e_{\mu}^{a}\right]=\int d^{4} x e \mathcal{L} \quad \text { with } \quad \mathcal{L} \equiv \frac{i}{2} \xi^{\dagger}\left(e_{a}^{\mu} \sigma^{a} \vec{D}_{\mu}-\overleftarrow{D}_{\mu} \sigma^{a} e_{a}^{\mu}\right) \xi, \quad e \equiv \operatorname{det}\left(e_{\mu}^{a}\right)
$$


where we introduced $\sigma^{a}=\left(1, \sigma^{i}\right)$ with the Pauli matrices $\sigma^{i}(i=1,2,3)$. Here $e_{\mu}^{a}\left(e_{a}^{\mu}\right)$ denotes (inverse) vierbein satisfying $g_{\mu \nu}=e_{\mu}^{a} e_{v}^{b} \eta_{a b}, \eta^{a b}=e_{\mu}^{a} e_{v}^{b} g^{\mu v}$ with the spacetime curved metric $g_{\mu \nu}$ and Minkowski metric $\eta_{a b}=\operatorname{diag}(-1,+1,+1,+1)$. The left and right covariant derivatives are defined as

$$
\vec{D}_{\mu} \xi \equiv \partial_{\mu} \xi-i\left(\mathcal{A}_{\mu}+A_{\mu}\right) \xi, \quad \xi^{\dagger} \overleftarrow{D}_{\mu} \equiv \partial_{\mu} \xi^{\dagger}+i \xi^{\dagger}\left(\mathcal{A}_{\mu}+A_{\mu}\right) \quad \text { with } \quad \mathcal{A}_{\mu} \equiv \frac{1}{2} \omega_{\mu}^{a b} \Sigma_{a b}
$$

where we introduced $\Sigma_{a b} \equiv i\left(\sigma^{a} \bar{\sigma}^{b}-\sigma^{b} \bar{\sigma}^{a}\right) / 4$ with $\bar{\sigma}^{a} \equiv\left(-1, \sigma^{i}\right)$, which satisfies $\sigma^{a} \bar{\sigma}^{b}+\sigma^{b} \bar{\sigma}^{a}=2 \eta^{a b}$. Furthermore, employing the torsionless condition, we can express the spin connection $\omega_{\mu}^{a b}=-\omega_{\mu}^{b a}$ as

$$
\omega_{\mu}^{a b} \equiv \frac{1}{2} e^{a v} e^{b \rho}\left(C_{v \rho \mu}-C_{\rho v \mu}-C_{\mu v \rho}\right) \quad \text { with } \quad C_{\mu v \rho} \equiv e_{\mu}^{c}\left(\partial_{\nu} e_{\rho c}-\partial_{\rho} e_{v c}\right) .
$$

Although the classical action (2) is invariant under a set of infinitesimal diffeomorphisms, local Lorentz, and $U(1)$ gauge transformations with parameters $\chi \equiv\left\{\zeta^{\mu}, \alpha_{a b}, \theta\right\}$ :

$$
\left\{\begin{array}{l}
\delta_{\chi} e_{\mu}^{a}=\zeta^{v} \nabla_{\nu} e_{\mu}^{a}+e_{\nu}^{a} \nabla_{\mu} \zeta^{v}+\alpha^{a}{ }_{b} e_{\mu}^{b}, \\
\delta_{\chi} A_{\mu}=\zeta^{v} \nabla_{\nu} A_{\mu}+A_{\nu} \nabla_{\mu} \zeta^{v}+\partial_{\mu} \theta, \\
\delta_{\chi} \xi=\zeta^{v} \partial_{\nu} \xi-\frac{i}{2} \alpha^{a b} \Sigma_{a b} \xi+i \theta \xi
\end{array}\right.
$$

we encounter with the quantum anomaly attached to the Weyl fermion. As a consequence, the anomalous Ward-Takahashi identities results in the following operator identities corresponding to the (non-)conservation laws:

$$
\left\{\begin{array}{l}
\nabla_{\mu} \hat{T}_{v}^{\mu}=F_{v \mu} \hat{J}^{\mu} \\
\nabla_{\mu} \hat{J}^{\mu}=-\frac{1}{8} C \varepsilon^{\mu v \rho \sigma} F_{\mu \nu} F_{\rho \sigma}-\lambda \varepsilon^{\mu v \rho \sigma} R_{\beta \mu \nu}^{\alpha} R_{\alpha \rho \sigma}^{\beta}
\end{array}\right.
$$

where we introduced the energy-momentum tensor $\hat{T}_{v}^{\mu}, U(1)$ covariant charge current $\hat{J}^{\mu}$ defined as

$$
\begin{aligned}
\hat{T}^{\mu v} & =\frac{1}{e} \frac{\delta S}{\delta e_{\mu}^{a}} e_{a}^{v}=-\frac{i}{2} \xi^{\dagger}\left(\sigma^{\mu} \overrightarrow{D^{v}}-\overleftarrow{D^{v}} \sigma^{\mu}\right) \xi+\frac{1}{4} D_{\rho}\left(\eta^{\dagger}\left\{\sigma^{\mu}, \Sigma^{v \rho}\right\} \eta\right)+\mathcal{L} g^{\mu \nu} \\
\hat{J}^{\mu} & =\frac{1}{e} \frac{\delta S}{\delta e_{\mu}^{a}}=\xi^{\dagger} \sigma^{\mu} \xi .
\end{aligned}
$$

The Lorentz invariance implies that the antisymmetric part of the energy tensor vanishes: $T^{\mu v}+T^{v \mu}=0$; thus, $T^{\mu v}$ can be regarded as symmetric one. We also defined a field strength tensor for the background electromagnetic field $F_{\mu \nu} \equiv \partial_{\mu} A_{\nu}-\partial_{\nu} A_{\mu}$, and the Riemann curvature tensor $R_{v \rho \sigma}^{\mu}$ with the totally antisymmetric tensor $\varepsilon^{\mu v \rho \sigma}$ satisfying $\varepsilon^{0123}=1 / e$. For notational simplicity, we drop the subscript $R$ for the $U(1)$ current. Here $C=1 /\left(4 \pi^{2}\right)$ and $\lambda=1 /\left(768 \pi^{2}\right)$ denote the anomaly coefficients coming from gauge and gravitational sectors, respectively. Since $\lambda \varepsilon^{\mu \nu \rho \sigma} R_{\beta \mu \nu}^{\alpha} R_{\alpha \rho \sigma}^{\beta}$ contains four derivatives, it does not contribute to the first-order hydrodynamics that we are interested in. Therefore, we will omit the gravitational part in the following discussion. Please note that while the gauge and diffeomorphism invariance provides two (non-)conservation laws, the local Lorentz invariance results in the symmetric property of the energy-momentum tensor operator. It is worth emphasizing that $\hat{J}^{\mu}$ in Equation (6) is the covariant current which can be related to the consistent current $\hat{J}_{\text {con }}^{\mu}$ by

$$
\hat{J}^{\mu}=\hat{J}_{\mathrm{con}}^{\mu}-\frac{1}{6} C \varepsilon^{\mu \nu \rho \sigma} A_{\nu} F_{\rho \sigma} .
$$

An analogue of this relation in local thermal equilibrium will appear in Section 4, and it plays an important role to see how the anomaly matching is realized for the local thermodynamic functional. 


\subsection{Zubarev's Formula: Decomposing Dissipative and Nondissipative Transport}

We then briefly review the Zubarev's nonequilibrium statistical operator method from the modern viewpoint (See e.g., Refs. [98-104] for recent discussions) and specify from where the anomaly-induced transport arises. Assuming that the system is initially in local thermal equilibrium, the Zubarev's formula provides us the expectation values of conserved current operators $\hat{\mathcal{J}}_{a}^{\mu} \equiv\left\{\hat{T}_{v}^{\mu}, \hat{J}^{\mu}\right\}$ over the initial density operator in the following compact form:

$$
\left\langle\hat{\mathcal{J}}_{a}^{\mu}(t, \boldsymbol{x})\right\rangle=\left\langle\hat{\mathcal{J}}_{a}^{\mu}(t, \boldsymbol{x})\right\rangle_{t}^{\mathrm{LG}}+L_{a b}^{\mu v}(t, \boldsymbol{x}) \nabla_{v} \lambda^{b}(t, x)+O\left((\nabla \lambda)^{2}\right),
$$

where we introduced the intensive local thermodynamic parameters $\lambda^{a} \equiv\left\{\beta^{\mu}, v\right\}$, which are related to the local fluid temperature $T=1 / \beta$, four-velocity $u^{\mu}$, and the chemical potential $\mu$ through $\beta^{\mu} \equiv \beta u^{\mu}$, $v \equiv \beta \mu$. We also defined the average over the local Gibbs distribution $\hat{\rho}_{\mathrm{LG}}[\lambda ; t]$, which describes systems in local thermal equilibrium, for an arbitrary operator $\hat{\mathcal{O}}$ as

$$
\langle\hat{\mathcal{O}}\rangle_{t}^{\mathrm{LG}} \equiv \operatorname{Tr}\left(\hat{\rho}_{\mathrm{LG}}[\lambda ; t] \hat{\mathcal{O}}\right) \quad \text { with } \quad \hat{\rho}_{\mathrm{LG}}[\lambda ; t] \equiv \exp [-\hat{S}[\lambda ; t]], \quad \hat{S}[\lambda ; t]=\hat{K}[\lambda ; t]+\Psi[\lambda ; t],
$$

where the entropy operator $\hat{S}[\lambda ; t]$ is composed of the part including operators $\hat{\mathcal{J}}_{a}^{\mu}$ and normalization part for the density operator:

$$
\begin{aligned}
& \hat{K}[\lambda ; t] \equiv-\int d \Sigma_{t \mu}\left[\beta^{v}(t, x) \hat{T}_{v}^{\mu}(t, x)+v(t, x) \hat{J}^{\mu}(t, x)\right], \\
& \Psi[\lambda ; t] \equiv \log \operatorname{Tr} \exp [-\hat{K}[\lambda ; t]] .
\end{aligned}
$$

We here employed the fully covariant notion by introducing the constant time (spacelike) hypersurface defined by its perpendicular surface vector $d \Sigma_{t \mu} \equiv-d^{3} x \sqrt{\gamma} n_{\mu}$. Choosing a certain globally defined time-coordinate function $\bar{t}(x)$, the unit normal vector $n_{\mu}$ can be expressed as

$$
n_{\mu}(x)=-N(x) \partial_{\mu} \bar{t}(x) \quad \text { with } \quad N(x) \equiv\left(-\partial^{\mu} \bar{t}(x) \partial_{\mu} \bar{t}(x)\right)^{-1 / 2},
$$

where $N(x)$ is a so-called Lapse function. In addition, introducing the spatial coordinate on the $\bar{x}$, we have the induced metric $\gamma_{\mu \nu}=g_{\mu \nu}+n_{\mu} n_{v}$ whose spatial part gives $\gamma \equiv \operatorname{det} \gamma_{\bar{i} \bar{j}}$ (See e.g., Refs. [102,103] for a detailed geometric setup). The introduction of the covariantized notion looks a little bit complicated, but one can always take the flat limit by setting $(\bar{t}(x), \bar{x}(x))=(t, x)$, which results in e.g. $\left.d \Sigma_{t \mu}\right|_{\text {flat }}=d^{3} x \delta_{\mu}^{0}$. Although it might be desirable to distinguish two coordinate systems defined by $(t, x)$ and $(\bar{t}, \bar{x})$, we will basically omit overline for the later one for notational simplicity since only $(\bar{t}, \bar{x})$-coordinate system is mainly used. The normalization part $\Psi[\lambda ; t]$ is the local thermodynamic functional called the Massieu-Planck functional, and plays a central role in Section 4.

The crucial point here is that by construction, we can identify the first term in the right-hand-side of Equation (9) as the nondissipative transport taking place in locally thermalized system, whereas the second term as the dissipative correction coming from the deviation from local thermal equilibrium. In other words, the formula (9) gives a way to decompose the non-dissipative and dissipative transport at least in the leading-order derivative expansion. The second term is proportional to the (local) thermodynamic forces $\nabla_{v} \lambda^{b}$, and coefficients in front of them are indeed specified as transport coefficients such as the bulk/shear viscosity, and conductivity. They are expressed by the two-point (Kubo) correlation function, which is nothing but the Green-Kubo formula for the transport coefficient [98-104]. On the other hand, nondissipative part is often assumed to be simply given by the usual constitutive relation for a perfect fluid. This is the case for parity-invariant systems, since the nondissipative derivative corrections are accompanied with higher-order derivatives for parity-invariant systems. Nevertheless, if we consider a system without parity symmetry-like the Weyl fermion system given in Equation (2)—we generally encounter with first-order nondissipative 
derivative corrections in $\left\langle\hat{\mathcal{J}}_{a}^{\mu}(t, x)\right\rangle_{t}^{\mathrm{LG}}$. This is the origin of the anomaly-induced transport, and we will focus on how we can evaluate $\left\langle\hat{\mathcal{J}}_{a}^{\mu}(t, x)\right\rangle_{t}^{\mathrm{LG}}$ in the remaining part of this paper.

Before closing this section, we put a short comment on the absence of the anomalous contribution to the entropy production. To see this, using the conservation laws (6), we express the entropy production operator $\hat{\Sigma}\left[t, t_{0} ; \lambda\right] \equiv \hat{S}[\lambda ; t]-\hat{S}\left[\lambda ; t_{0}\right]$ as

$$
\hat{\Sigma}\left[t, t_{0} ; \lambda\right]=\int_{t_{0}}^{t} d^{4} x e \nabla_{\mu} \hat{s}^{\mu} \quad \text { with } \quad \nabla_{\mu} \hat{s}^{\mu} \equiv-\left(\nabla_{v} \beta^{\mu}\right) \delta \hat{T}_{v}^{\mu}-\left(\nabla_{\mu} v+\beta^{v} F_{\mu v}\right) \delta \hat{J}^{\mu},
$$

where we defined the local entropy production rate $\nabla_{\mu} \hat{s}^{\mu}$ with $\delta \mathcal{O}(t) \equiv \mathcal{O}(t)-\langle\mathcal{O}(t)\rangle_{t}^{\mathrm{LG}}$. We thus find that the local equilibrium part of the constitutive relation $\left\langle\hat{\mathcal{J}}_{a}^{\mu}\right\rangle_{t}^{\mathrm{LG}}$ which also contains the anomaly-induced transport as first-order derivative corrections, does not contribute to the local entropy production. This is perfectly consistent with the phenomenological derivation of the anomaly-induced transport based on the entropy-current analysis given in Ref. [24].

\section{Perturbative Evaluation of Anomalous Transport Coefficients}

In this section, we provide a simple perturbative derivation of the anomaly-induced transport given in Equation (1), and calculate anomalous transport coefficients $\sigma_{B}$ and $\sigma_{\omega}$ at the one-loop level.

\subsection{Derivative Expansion of the Local Gibbs Distribution}

First of all, we note that the local equilibrium part of the constitutive relation, or $\left\langle\hat{\mathcal{J}}_{a}^{\mu}(t, x)\right\rangle_{t}^{\mathrm{LG}}$, is a functional of local thermodynamic parameters $\lambda^{a}=\left\{\beta^{\mu}, \nu\right\}$ and external fields $j \equiv\left\{A_{\mu}, e_{\mu}^{a}\right\}$ at a fixed constant time $t$ since the local Gibbs distribution $\hat{\rho}_{\mathrm{LG}}[\lambda ; t]$ depends on the configuration of them. Thus, $\left\langle\hat{\mathcal{J}}_{a}^{\mu}(t, x)\right\rangle_{t}^{\mathrm{LG}}$ inherently contains the derivative correction coming from the local Gibbs distribution itself.

Suppose that our system is described by the local Gibbs distribution slightly deviated from the global equilibrium (Gibbs) distribution only with the magnetic field and fluid vorticity. We also turn off the external fields and take the flat limit. In that situation, approximating the fluid velocity and the magnetic field as

$$
\left\{\begin{array}{l}
u_{j}(x)=\left.\left(x^{i}-x_{0}^{i}\right) \partial_{i} u_{j}\right|_{x=x_{0}}=\left(x^{i}-x_{0}^{i}\right) \epsilon_{i j k} \omega^{k}, \\
A_{j}(x)=\left.\left(x^{i}-x_{0}^{i}\right) \partial_{i} A_{j}\right|_{x=x_{0}}=\frac{1}{2}\left(x^{i}-x_{0}^{i}\right) \epsilon_{i j k} B^{k},
\end{array}\right.
$$

we can expand the local Gibbs distribution on the top of the global Gibbs distribution as

$$
\hat{\rho}_{\mathrm{LG}}[\lambda ; t]=\frac{1}{Z} e^{-\beta(\hat{H}-\mu \hat{N})}\left[1+T_{\tau} \int_{0}^{\beta} d \tau \Delta \hat{S}(t-i \tau)\right] \quad \text { with } \quad \Delta \hat{S} \equiv \frac{1}{2} \int d^{3} x \epsilon_{i j k}\left(x^{i}-x_{0}^{i}\right)\left(\hat{J}^{j} B^{k}+2 \hat{T}^{0 j} \omega^{k}\right),
$$

where we defined $\hat{\mathcal{O}}(t-i \tau) \equiv e^{\tau(\hat{H}-\mu \hat{N})} \hat{\mathcal{O}}(t) e^{-\tau(\hat{H}-\mu \hat{N})}$. Here $Z \equiv \operatorname{Tr} e^{-\beta(\hat{H}-\mu \hat{N})}$ denotes the partition function for the globally thermalized system, and we use $\langle\hat{\mathcal{O}}\rangle_{\text {eq }} \equiv \operatorname{Tr}\left(e^{-\beta(\hat{H}-\mu \hat{N})} \hat{\mathcal{O}}\right) / Z$. Then, noting that the averaged current in global thermal equilibrium vanishes $\left\langle\hat{J}^{i}\left(t, x_{0}\right)\right\rangle_{\mathrm{eq}}=0$, we can evaluate $\left\langle\hat{J}^{i}\left(t, x_{0}\right)\right\rangle_{t}^{\mathrm{LG}}$ as

$$
\begin{aligned}
\left\langle\hat{J}^{i}\left(t, x_{0}\right)\right\rangle_{t}^{\mathrm{LG}}= & \frac{1}{2} \int_{0}^{\beta} d \tau \int d^{3} x \epsilon_{j k l}\left(x^{j}-x_{0}^{j}\right) \\
& \times\left[\left\langle\hat{J}^{k}(t-i \tau, \boldsymbol{x}) \hat{J}^{i}\left(t, x_{0}\right)\right\rangle_{\mathrm{eq}} B^{l}\left(t, x_{0}\right)+2\left\langle\hat{T}^{0 k}(t-i \tau, \boldsymbol{x}) \hat{J}^{i}\left(t, x_{0}\right)\right\rangle_{\mathrm{eq}} \omega^{l}\left(t, x_{0}\right)\right] \\
= & \frac{i}{2} \epsilon_{j k l}\left[\left.\partial_{q_{j}} \Delta_{J^{k} j^{i}}\left(\omega_{n}, \boldsymbol{q}\right)\right|_{\omega_{n}=0, \boldsymbol{q}=\mathbf{0}} B^{l}\left(t, x_{0}\right)+\left.2 \partial_{q_{j}} \Delta_{T^{0 k} j^{i}}\left(\omega_{n}, \boldsymbol{q}\right)\right|_{\omega_{n}=0, \boldsymbol{q}=0} \omega^{l}\left(t, \boldsymbol{x}_{0}\right)\right],
\end{aligned}
$$

where we performed the Fourier transformation to proceed the second line. It is now clear that we only need to evaluate two-point imaginary-time-not real-time-correlation functions, namely $\left\langle\hat{J}^{k}(t-i \tau, x) \hat{J}^{i}\left(t, x_{0}\right)\right\rangle_{\mathrm{eq}}$ and $\left\langle\hat{T}^{0 k}(t-i \tau, x) \hat{J}^{i}\left(t, x_{0}\right)\right\rangle_{\mathrm{eq}}$, or their low-frequency and wave-number in the Fourier space. 


\subsection{One-loop Evaluation of Anomalous Transport Coefficients}

We then evaluate the anomalous transport coefficients with the help of the Matsubara formalism. Since we expand the local Gibbs distribution on the top of global Gibbs distribution, the Euclidean action $S_{E}\left[\xi, \xi^{\dagger}, \mu\right]$ for the right-handed Weyl fermion is simply given by

$$
S_{E}\left[\xi, \xi^{\dagger}\right]=-\sum_{P} \xi_{a}^{\dagger}(P)\left(\mathcal{G}_{0}^{-1}(\tilde{P})\right)_{a b} \xi_{b}(P) \quad \text { with } \quad \mathcal{G}_{0}^{-1}(P) \equiv \sigma^{\mu} P_{\mu}, \quad \mathcal{G}_{0}(P)=\frac{\bar{\sigma}^{\mu} P_{\mu}}{P^{2}},
$$

where $a, b(=1,2)$ denote the spinor indices, and $\mathcal{G}_{0}(P)$ the free propagator for the Weyl fermion. We also defined $\tilde{P}_{\mu} \equiv\left(-i \omega_{n}-\mu, p\right)$ with the Matsubara frequency $\omega_{n} \equiv(2 n+1) \pi T$ and chemical potential $\mu$. As usual, we introduced the Fourier transformation

$$
\xi(\tau, x)=T \sum_{n} \int \frac{d^{3} \boldsymbol{p}}{(2 \pi)^{3}} e^{-i \omega_{n} \tau+i p \cdot x} \xi\left(\omega_{n}, \boldsymbol{p}\right),
$$

with the temperature $T \equiv 1 / \beta$. Please note that the argument of the propagator in Equation (18) is not $P$ but $\tilde{P}$, and, thus, it represents the propagator fully dressed by the chemical potential $\mu$. By using these, we need to evaluate the following diagrams:

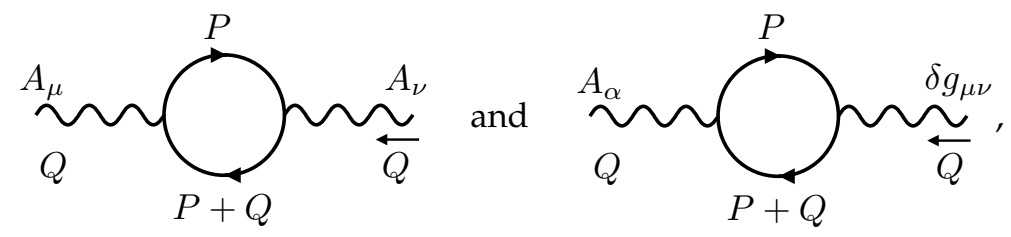

where we will take the long-wave-length limit $Q \sim 0$.

First, let us evaluate the two-point current-current correlation function given by

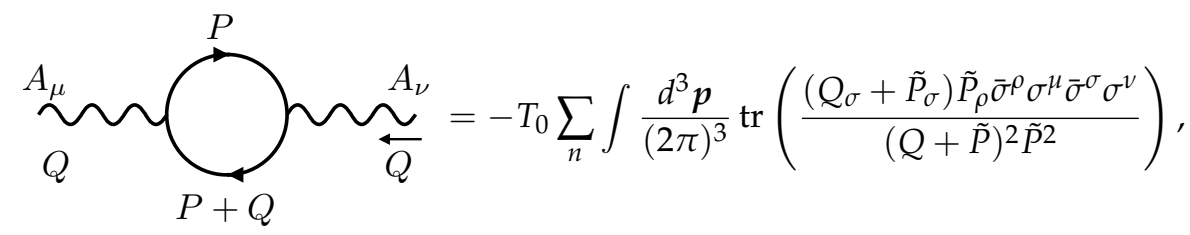

where we used the free propagator defined in Equation (18). Here " $\operatorname{tr}$ " denotes the trace over the spinor indices. With the help of the trace formula for the Pauli matrices

$$
\operatorname{tr} \bar{\sigma}^{\mu} \sigma^{\nu} \bar{\sigma}^{\alpha} \sigma^{\beta}=-2 i \varepsilon^{\mu \nu \alpha \beta}+2 \eta^{\mu \nu} \eta^{\alpha \beta}-2 \eta^{\mu \alpha} \eta^{\nu \beta}+2 \eta^{\mu \beta} \eta^{v \alpha},
$$

we can decompose the two-point functions into the antisymmetric part and other parts. Since we are interested in the anomalous term which results from the antisymmetric part, we only focus on that part:

$$
\overbrace{Q}^{A_{\mu}} \overbrace{P+Q}^{P} \overbrace{\frac{A_{\nu}}{(2}}^{A_{L}}=-\frac{i \mu}{4 \pi^{2}} \varepsilon^{0 \mu \nu \rho} Q_{\rho}+(\text { symmetric terms })+O\left(Q^{2}\right),
$$


Next, let us evaluate the two-point momentum-current correlation function. Then, the same calculus brings about the following result

$$
\begin{aligned}
& \sim_{Q}^{A_{\alpha}} \overbrace{P+Q}^{P} \overbrace{Q}^{\delta g_{\mu \nu}}=-\frac{1}{4} T_{0} \sum_{n} \int \frac{d^{3} p}{(2 \pi)^{3}}\left(2 \tilde{P}^{\gamma}+Q^{\gamma}\right)\left(\delta_{\beta}^{\mu} \delta_{\gamma}^{\nu}+\delta_{\beta}^{\nu} \delta_{\gamma}^{\mu}\right) \operatorname{tr}\left(\frac{\left(Q_{\sigma}+\tilde{P}_{\sigma}\right) \tilde{P}_{\rho} \bar{\sigma}^{\rho} \sigma^{\beta} \bar{\sigma}^{\sigma} \sigma^{\alpha}}{(Q+\tilde{P})^{2} \tilde{P}^{2}}\right) \\
& =i Q_{\rho}\left(\eta^{\nu 0} \mathcal{E}^{\rho \mu 0 \alpha}+\eta^{\mu 0} \mathcal{E}^{\rho v 0 \alpha}+\delta_{j}^{v} \varepsilon^{\rho \mu j \alpha}+\delta_{j}^{\mu} \varepsilon^{\rho v j \alpha}\right)\left(\frac{\mu^{2}}{16 \pi^{2}}+\frac{T_{0}^{2}}{48}\right) \\
& +(\text { symmetric terms })+\mathcal{O}\left(Q^{2}\right) \text {. }
\end{aligned}
$$

Putting these results all together, Equation (17) results in

$$
\left\langle\hat{J}^{i}\left(t, x_{0}\right)\right\rangle_{t}^{\mathrm{LG}}=\frac{\mu}{4 \pi^{2}} B^{i}\left(t, x_{0}\right)+\left(\frac{\mu^{2}}{4 \pi^{2}}+\frac{T^{2}}{12}\right) \omega^{i}\left(t, x_{0}\right),
$$

which is nothing but Equation (1). To summarize the above analysis, we have derived the anomaly-induced transport-chiral magnetic/vortical effect-for the Weyl fermion by expanding the local Gibbs distribution. This clearly shows that information on the anomaly-induced transport is fully contained in $\left\langle\hat{\mathcal{J}}_{a}^{\mu}(t, x)\right\rangle_{t}^{\mathrm{LG}}$. Although we performed the direct expansion of the local Gibbs distribution in this section, there is another way to systematically evaluate $\left\langle\hat{\mathcal{J}}_{a}^{\mu}(t, x)\right\rangle_{t}^{\mathrm{LG}}$ as we will see in the next section.

\section{Anomaly Matching for Local Thermodynamic Functional}

In the previous section, we have explicitly shown that the local equilibrium part of constitutive relations $\left\langle\hat{\mathcal{J}}_{a}^{\mu}(t, x)\right\rangle_{t}^{\mathrm{LG}}$ indeed contains the information on the anomaly-induced transport. Although it is the one-loop perturbative calculation, we expect the result, or the value of anomalous transport coefficients, is protected by the underlying chiral anomaly, and remain the same even if we take into account the effect of interactions nonperturbatively. In this section, we provide another way to see the anomaly-induced transport putting the emphasis on the nonperturbative aspect of the anomaly. The key quantity is the local thermodynamic functional $\Psi[\lambda, j ; t]$ already defined in Equation (12).

\subsection{Basic Properties of Local Thermodynamic Functional}

We here summarize basic properties of the Massieu-Planck functional $\Psi[\lambda, j ; t]$ : the exact path-integral expression of $\Psi[\lambda, j ; t]$ and resulting symmetry properties together with the variational formula.

\subsubsection{Path-Integral Formula and Resulting Symmetry}

We will first summarize the key result for the Massieu-Planck functional (See Refs. [102,103] for the derivation). Using the energy-momentum tensor operator $\hat{T}_{v}^{\mu}$ and covariant current operator $\hat{j}^{\mu}$ resulting from (2), we can express the Massieu-Planck functional by the imaginary-time path-integral in the same way with the usual Matsubara formalism for global thermal equilibrium. After a little bit tedious calculation (See Ref. [103]), we eventually obtain

$$
\Psi[\lambda, j ; t]=\int \mathcal{D} \xi \mathcal{D} \xi^{\dagger} \exp \left(\widetilde{\mathcal{S}}\left[\xi, \xi^{\dagger} ; \widetilde{A}, \widetilde{e}\right]\right),
$$

with the manifestly covariant action $\widetilde{\mathcal{S}}\left[\xi, \xi^{+} ; \widetilde{A}_{\mu}, \widetilde{e}_{\mu}^{a}\right]$ given by

$$
\widetilde{\mathcal{S}}\left[\xi, \xi^{\dagger} ; \widetilde{A}_{\mu}, \widetilde{e}_{\mu}^{a}\right]=\int_{0}^{\beta_{0}} d \tau d^{3} x \widetilde{e}\left[\frac{i}{2} \xi^{\dagger}\left(\widetilde{e}_{a}^{\mu} \sigma^{a} \overrightarrow{\widetilde{D}}_{\mu}-\overleftarrow{D}_{\mu} \sigma^{a} \widetilde{e}_{a}^{\mu}\right) \xi\right] \quad \text { with } \quad \widetilde{e} \equiv \operatorname{det}\left(\widetilde{e}_{\mu}^{a}\right)
$$


Here we introduced the thermal (inverse) vierbein $\widetilde{e}_{\mu}^{a}\left(\widetilde{e}_{a}^{\mu}\right)$ and the external $U(1)$ gauge field $\widetilde{A}_{\mu}$ in thermally emergent curved spacetime as

$$
\widetilde{e}_{0}^{a}=e^{\sigma} u^{a}, \quad \widetilde{e}_{i}^{a}=e_{i}^{a} \quad \text { and } \quad \widetilde{A}_{0}=e^{\sigma} \mu, \quad \widetilde{A}_{i}=A_{i},
$$

where recalling $\beta^{\mu}(x) \equiv \beta(x) u^{\mu}(x)$ and $v(x)=\beta(x) \mu(x)$, we used

$$
e^{\sigma(x)} \equiv \beta(x) / \beta_{0}, \quad \mu(x) \equiv v(x) / \beta(x), \quad \beta(x) \equiv \sqrt{-g_{\mu v}(x) \beta^{\mu}(x) \beta^{v}(x)},
$$

with a constant reference inverse temperature $\beta_{0}$. We also introduced $\widetilde{e} \equiv \operatorname{det} \widetilde{e}_{\mu}^{a}$ and the covariant derivative in thermal spacetime as

$$
\left\{\begin{array}{l}
\overrightarrow{\widetilde{D}}_{\mu} \xi \equiv \widetilde{\partial}_{\mu} \xi-i\left(\widetilde{\mathcal{A}}_{\mu}+\widetilde{A}_{\mu}\right) \xi \\
\xi^{+} \overleftarrow{D}_{\mu} \equiv \widetilde{\partial}_{\mu} \xi^{\dagger}+i \xi^{\dagger}\left(\widetilde{\mathcal{A}}_{\mu}+\widetilde{A}_{\mu}\right)
\end{array} \quad \text { with } \quad \widetilde{\partial}_{\mu} \equiv\left(i \partial_{\tau}, \partial_{i}\right), \quad \widetilde{\mathcal{A}}_{\mu} \equiv \frac{1}{2} \widetilde{\omega}_{\mu}^{a b} \Sigma_{a b}\right.
$$

where the thermal spin connection is expressed by the thermal vierbein $\widetilde{e}_{\mu}^{a}$ through the same relation in the original spacetime (4).

As is shown in these, we can say that the Massieu-Planck functional is expressed as the path-integral in the presence of the emergent background curved spacetime and $U(1)$ gauge field. Figure 2 shows a schematic picture to compare the imaginary-time formalism in global and local thermal equilibrium.

(a) Global thermal equilibrium

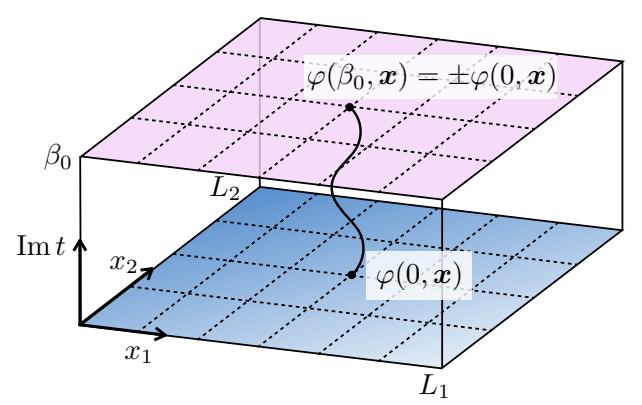

(b) Local thermal equilibrium

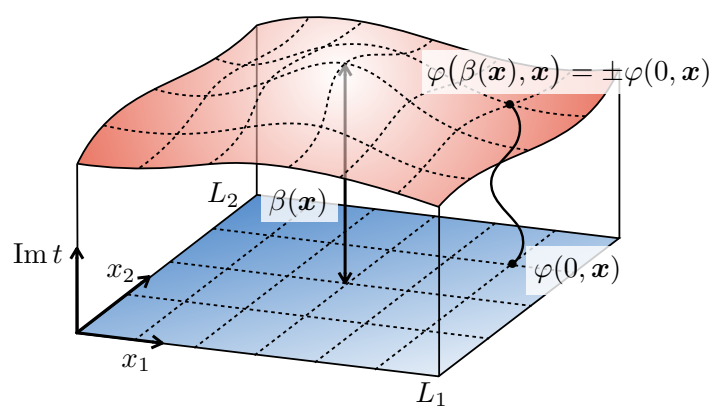

Figure 2. A comparison of the imaginary-time formalism (a) in global thermal equilibrium and (b) in local thermal equilibrium. Effects of inhomogeneous local thermodynamic parameters such as the local temperature is completely captured in terms of the emergent curved geometry. The boundary condition \pm corresponds to the boson or the fermion.

Please note that this background structure is completely determined by configurations of the local thermodynamic variables $\lambda^{a}$ (and external fields $j$ ) on the constant time hypersurface in the original spacetime. The crucial point here is that all these quantities do not depend on the imaginary-time coordinate $\tau$, which leads to the Kaluza-Klein gauge symmetry. To see this clearly, we express the line element $d \widetilde{s}^{2} \equiv \widetilde{e}_{\mu}^{a} \widetilde{e}_{v}^{b} \eta_{a b} d \widetilde{x}^{\mu} \otimes d \widetilde{x}^{v}$ and $U(1)$ gauge connection $\widetilde{A} \equiv \widetilde{A}_{\mu} d \widetilde{x}^{\mu}$ in thermal spacetime as

$$
\begin{aligned}
d \widetilde{s}^{2} & =-e^{2 \sigma}\left(d \widetilde{t}+a_{i} d x^{i}\right)^{2}+\gamma_{i j}^{\prime} d x^{i} d x^{j}, \\
\widetilde{A} & =\widetilde{A}_{0}\left(\widetilde{d t}+a_{i} d x^{i}\right)+\widetilde{A}_{i}^{\prime} d x^{i},
\end{aligned}
$$

with $d \widetilde{t} \equiv-i d \tau$. Here we defined the following quantities

$$
a_{i} \equiv-e^{-\sigma} u_{i}, \quad \gamma_{i j}^{\prime} \equiv \gamma_{i j}+e^{2 \sigma} a_{i} a_{j}, \quad \widetilde{A}_{i}^{\prime} \equiv=\widetilde{A}_{i}-\widetilde{A}_{0} a_{i} .
$$


Then, in addition to the spatial diffeomorphism invariance-invariance under spatial coordinate transformation $x \rightarrow x^{\prime}(x)$ - we now see the background (30) and (31) is invariant under the transformation given by

$$
\left\{\begin{array}{l}
\tilde{t} \rightarrow \widetilde{t}+\chi(x) \\
x \rightarrow x \\
a_{i}(x) \rightarrow a_{i}(x)-\partial_{i} \chi(x)
\end{array}\right.
$$

This is nothing but Kaluza-Klein gauge transformation, and $a_{i}$ is identified as the Kaluza-Klein gauge field. Please note that $\gamma_{i j}$ and $\widetilde{A}_{i}=A_{i}$ do transform under the Kaluza-Klein gauge transformation so that $\gamma_{i j}^{\prime}$ and $\widetilde{A}_{i}^{\prime}$ do not. Therefore, it is useful to employ Kaluza-Klein gauge invariant quantities $\gamma_{i j}^{\prime}$ and $\widetilde{A}_{i}^{\prime}$ rather than $\gamma_{i j}$ and $\widetilde{A}_{i}$ as basic building blocks to construct the Massieu-Planck functional. Furthermore, since the system is composed of the Weyl fermion, the apparent $U(1)$ gauge invariance for $\widetilde{A}_{i}^{\prime}$ is anomalously broken. These spatial diffeomorphism, Kaluza-Klein gauge, and anomalous $U(1)$ gauge symmetries provide a basic restriction to the Massieu-Planck functional.

\subsubsection{Variational Formula in the Presence of Quantum Anomaly}

We then provide the variational formula for the Massieu-Planck functional $\Psi[\lambda, j ; t]$, and show all information on $\left\langle\hat{\mathcal{J}}_{a}^{\mu}(t, x)\right\rangle_{t}^{\mathrm{LG}}$ is fully installed in it. To show this, let us consider the variation of $\hat{K}$ defined in Equation (11) under the infinitesimal general coordinate and gauge transformation with a set of parameters $\zeta^{\mu}=\epsilon \beta^{\mu}$ and $\theta=\epsilon(\nu-\beta \cdot A)$. ( $\epsilon$ denotes an infinitesimal constant.) As a result, of the combination of diffeomorphism and $U(1)$ gauge transformations, the variation of the background $U(1)$ gauge field $\delta_{\lambda} A_{\mu}$ has the simple expression:

$$
\delta_{\lambda} A_{\mu}=£_{\beta} A_{\mu}+\nabla_{\mu}(v-\beta \cdot A)=\nabla_{\mu} v+\beta^{\nu} F_{\nu \mu} .
$$

The crucial point here is that $\hat{K}$ remains invariant under the simultaneous transformation acting on both operators and external fields: $\delta_{\lambda} \hat{K} \equiv \delta_{\lambda}^{\text {para }} \hat{K}+\delta_{\lambda}^{\text {ope }} \hat{K}=0$. This invariance can be shown by recalling all operators in $\hat{K}$ are $U(1)$ gauge invariant, and, furthermore, rewriting $\hat{K}$ as

$$
\hat{K}\left[t, \lambda^{a}, e_{\mu}^{a}, A_{\mu}\right]=\int d^{4} x \sqrt{\gamma} \delta(t-t(x)) n_{\mu}(x) \lambda^{a}(x) \hat{\mathcal{J}}_{a}^{\mu}(x),
$$

from which we can clearly see diffeomorphism (reparameterization) invariance. Moreover, $\delta_{\lambda}^{\text {ope }} \hat{K}$ will also trivially vanish just because $\delta_{\lambda}^{\text {ope }} \hat{K}=[i \hat{K}, \hat{K}]=0$. As a result, we have the operator identity $\delta_{\lambda}^{\text {para }} \hat{K}=0$.

Then, let us investigate $\delta_{\lambda}^{\text {para }} \hat{K}$ in detail, whose explicit definition is given by

$$
\delta_{\lambda}^{\mathrm{para}} \hat{K} \equiv \int d^{4} x\left[\frac{\delta \hat{K}}{\delta t(x)} £_{\beta} t(x)+\frac{\delta \hat{K}}{\delta \lambda^{a}(x)} £_{\beta} \lambda^{a}(x)+\frac{\delta \hat{K}}{\delta e_{\mu}^{a}(x)} £_{\beta} e_{\mu}^{a}(x)+\frac{\delta \hat{K}}{\delta A_{\mu}(x)} \delta_{\lambda} A_{\mu}(x)\right] .
$$

To rewrite the first term of this equation, noting $\delta(t-t(x)) n_{\mu}=-N \delta(t-t(x)) \partial_{\mu} t=N \partial_{\mu} \theta(t-$ $t(x))$ following from the definition of $n_{\mu}$, and performing the integration by parts, we rewrite $\hat{K}$ in Equation (35) as

$$
\begin{aligned}
\hat{K}\left[t, \lambda^{a}, e_{\mu}^{a}, A_{\mu}\right] & =-\int d^{4} x e \theta(t-t(x)) \nabla_{\mu}\left(\lambda^{a}(x) \hat{\mathcal{J}}_{a}^{\mu}(x)\right) \\
& =-\int d^{4} x e \theta(t-t(x))\left(\hat{T}^{\mu} \nabla_{\mu} \beta^{v}+\hat{J}^{\mu}\left(\nabla_{\mu} v+\beta^{v} F_{\nu \mu}\right)-\frac{1}{8} C v \varepsilon^{\mu \nu \rho \sigma} F_{\mu v} F_{\rho \sigma}\right),
\end{aligned}
$$


where we used $e=N \sqrt{\gamma}$ and employed the operator identity for current operators (6) to proceed the second line. With the help of Equation (34) together with $\nabla_{\mu} \beta^{v}=e_{a}^{v} \varepsilon_{\beta} e_{\mu}^{a}+\beta^{\rho} \omega_{\rho \mu}^{v}$ followed from the so-called (torsionless) tetrad postulate $\nabla_{\mu} e_{v}^{a}+\omega_{\mu b}^{a} e_{v}^{b}=0$, Equation (37) enables us to obtain

$$
\int d^{4} x \frac{\delta \hat{K}}{\delta t(x)} £_{\beta} t(x)=\int d^{4} x \sqrt{\gamma} \delta(t-t(x))\left[\hat{T}_{a}^{\mu} £_{\beta} e_{\mu}^{a}+\hat{J}^{\mu} \delta_{\lambda} A_{\mu}-\frac{1}{8} C \nu \varepsilon^{\mu \nu \rho \sigma} F_{\mu \nu} F_{\rho \sigma}\right] \beta^{\prime},
$$

where we defined $\beta^{\prime} \equiv-\beta^{\mu} n_{\mu}$ and used the operator identity $\hat{T}^{a b}-\hat{T}^{b a}=0$. By using the identity

$$
n_{\alpha} \varepsilon^{\mu \nu \rho \sigma} F_{\mu v} F_{\rho \sigma}=-4 \varepsilon^{\mu v \rho \sigma} n_{v} F_{\rho \sigma} F_{\alpha \mu} .
$$

the last term in the second line of Equation (38) can be further simplified as

$$
\begin{aligned}
\int d^{4} x \sqrt{\gamma} \delta(t-t(x))\left[\frac{1}{8} C \nu \beta^{\alpha} n_{\alpha} \varepsilon^{\mu \nu \rho \sigma} F_{\mu v} F_{\rho \sigma}\right] & =-\int d^{4} x \sqrt{\gamma} \delta(t-t(x))\left[\frac{1}{2} C \nu \beta^{\alpha} n_{v} \varepsilon^{\mu v \rho \sigma} F_{\rho \sigma} F_{\alpha \mu}\right] \\
& =-\int d^{4} x \sqrt{\gamma} \delta(t-t(x)) C \nu B^{\mu} \delta_{\lambda} A_{\mu} .
\end{aligned}
$$

Here we defined the four-magnetic field as $B^{\mu} \equiv \widetilde{F}^{\mu v} n_{v}=\varepsilon^{\mu v \rho \sigma} n_{v} F_{\rho \sigma} / 2$, and neglected the surface term accompanied by the integration by parts. We thus obtain the following compact result:

$$
\int d^{4} x \frac{\delta \hat{K}}{\delta t(x)} £_{\beta} t(x)=\int d^{4} x \beta^{\prime} \sqrt{\gamma} \delta(t-t(x))\left[\hat{T}_{a}^{\mu} £_{\beta} e_{\mu}^{a}+\left(\hat{J}^{\mu}-C \beta^{\prime-1} v B^{\mu}\right) \delta_{\lambda} A_{\mu}\right] .
$$

Equipped with this formula together with $£_{\beta} \beta^{\mu}=0$, and $£_{\beta} v=£_{\beta}(v-\beta \cdot A)+\beta^{\mu} £_{\beta} A_{\mu}=\beta^{\mu} \delta_{\lambda} A_{\mu}$, we are now ready to express $\delta_{\lambda}^{\text {para }} \hat{K}$ in Equation (36) by the use of the variation of the vierbein and gauge field:

$$
\delta_{\lambda}^{\mathrm{para}} \hat{\mathrm{K}}=\int d^{3} x\left[\left(\beta^{\prime} \sqrt{\gamma} \hat{T}_{a}^{\mu}+\frac{\delta \hat{\mathrm{K}}}{\delta e_{\mu}^{a}}\right) £_{\beta} e_{\mu}^{a}+\left(\beta^{\prime} \sqrt{\gamma}\left[\hat{J}^{\mu}-C \beta^{\prime-1} v B^{\mu}\right]+\frac{\delta \hat{K}}{\delta v} \beta^{\mu}+\frac{\delta \hat{K}}{\delta A_{\mu}}\right) \delta_{\lambda} A_{\mu}\right] .
$$

Let us then take the average of this operator identity over the local Gibbs distribution $\hat{\rho}_{\mathrm{LG}}[\lambda ; t]$. In the absence of the quantum anomaly, we can simply replace the averaged variation of $\hat{K}$ with the variation of the Massieu-Planck functional: $\langle\delta \hat{K} / \delta j\rangle_{t}^{\mathrm{LG}}=-\delta \Psi / \delta j$. Nevertheless, since we are considering the system with the chiral anomaly, we need to be careful when we take the variation of the charge density coupled to the local chemical potential. Using the relation $\partial\left(e \hat{J}^{0}\right) / \partial A_{\mu}=\sqrt{\gamma} C B^{\mu}$ resulting from the covariant anomaly, we can show

$$
\frac{\delta \hat{K}}{\delta A_{\mu}}=e \hat{J}^{0} \beta^{\mu}+\sqrt{\gamma} \nu C B^{\mu}-\beta^{\prime} \sqrt{\gamma} \frac{\partial \hat{\mathcal{L}}}{\partial A_{\mu}} .
$$

We can then identify the local Gibbs average of the last term in this equation as the covariant current in thermal spacetime, which results in the sum of the consistent current and the Bardeen-Zumino current composed of $\widetilde{A}_{\mu}$ :

$$
\beta^{\prime} \sqrt{\gamma}\left\langle\frac{\partial \hat{\mathcal{L}}}{\partial A_{\mu}}\right\rangle_{t}^{\mathrm{LG}}=\mathcal{N} \int \mathcal{D} \xi \mathcal{D} \xi^{\dagger} e^{\widetilde{\mathcal{S}}\left[\xi, \xi^{\dagger} ; \widetilde{A}, \widetilde{e}\right]} \frac{\delta \widetilde{\mathcal{S}}}{\delta \widetilde{A}_{\mu}}=\frac{\delta \Psi}{\delta \widetilde{A}}-\frac{C}{6} \widetilde{\mathcal{E}}^{\mu v \rho \sigma} \widetilde{A}_{\nu} \mathcal{F}_{\rho \sigma},
$$

where $\mathcal{N}$ denotes a normalization constant, and we introduced a field strength tensor in thermal spacetime $\mathcal{F}_{\mu v} \equiv \widetilde{\partial}_{\mu} \widetilde{A}_{v}-\widetilde{\partial}_{v} \widetilde{A}_{\mu}$ together with the totally antisymmetric tensor $\widetilde{\mathcal{E}}^{\mu v \rho \sigma} \equiv N\left(\beta_{0} / \beta^{\prime}\right) \varepsilon^{\mu v \rho \sigma}$. Using this together with $\left\langle\delta \hat{K} / \delta e_{\mu}^{a}\right\rangle_{t}^{\mathrm{LG}}=-\delta \Psi / \delta e_{\mu}^{a}$, we eventually obtain the following identity: 


$$
\left\langle\delta_{\lambda}^{\mathrm{para}} \hat{\mathrm{K}}\right\rangle_{t}^{\mathrm{LG}}=\int d^{3} x\left[\left(\beta^{\prime} \sqrt{\gamma}\left\langle\hat{T}_{a}^{\mu}\right\rangle_{t}^{\mathrm{LG}}-\frac{\delta \Psi}{\delta e_{\mu}^{a}}\right) E_{\beta} e_{\mu}^{a}+\left(\beta^{\prime} \sqrt{\gamma}\left\langle\hat{J}^{\mu}\right\rangle_{t}^{\mathrm{LG}}-\frac{\delta \Psi}{\delta \tilde{A}_{\mu}}+\frac{C}{6} \widetilde{\mathcal{E}}^{u v \rho \sigma} \widetilde{A}_{\nu} \mathcal{F}_{\rho \sigma}\right) \delta_{\lambda} A_{\mu}\right] .
$$

Therefore, noting that that this identity holds for an arbitrary variation of the background vierbein and gauge field, the identity $\left\langle\delta_{\lambda}^{\text {para }} \hat{K}\right\rangle_{t}^{\mathrm{LG}}=0$ provides the variational formula for the Masseiu-Planck functional

$$
\begin{aligned}
\left\langle\hat{T}_{a}^{\mu}(t, x)\right\rangle_{t}^{\mathrm{LG}} & =\frac{1}{\beta^{\prime} \sqrt{\gamma}} \frac{\delta \Psi[\lambda, j, t]}{\delta e_{\mu}^{a}(x)}, \\
\left\langle\hat{J}^{\mu}(t, x)\right\rangle_{t}^{\mathrm{LG}} & =\frac{1}{\beta^{\prime} \sqrt{\gamma}} \frac{\delta \Psi[\lambda, j, t]}{\delta \widetilde{A}_{\mu}(x)}-\frac{C}{6} \widetilde{\varepsilon}^{\mu v \rho \sigma} \widetilde{A}_{v} \mathcal{F}_{\rho \sigma} .
\end{aligned}
$$

We thus conclude that the average values of any conserved current operator over local thermal equilibrium is fully captured by the single (local thermodynamic) functional known as the Masseiu-Planck functional. It is worth pointing out that because we deal with the average of the covariant current $\langle\hat{J}(x)\rangle_{t}^{\mathrm{LG}}$, we have the last term in Equation (47) analogous to the Bardeen-Zumino current [105] (See also Refs. [29,43,47]). In summary, we can identify the Massieu-Planck functional $\Psi[\lambda, j ; t]$ as a generating functional for a (nondissipative) local equilibrium part of hydrodynamics, or $\left\langle\hat{\mathcal{J}}_{a}^{\mu}(t, x)\right\rangle_{t}^{\mathrm{LG}}$.

Before moving to the path-integral formula for the Massieu-Planck functional, we put a short comment on the useful "gauge and coordinate choice", which we call hydrostatic gauge. Since we have a freedom to choose the local time-direction and time-component of the external gauge field, we can employ the hydrostatic gauge fixing condition

$$
t^{\mu}(x)=\beta^{\mu}(x) / \beta_{0}, \quad t^{\mu}(x) A_{\mu}(x)=v(x) / \beta_{0},
$$

with a constant reference temperature $\beta_{0}$. In this special choice of the gauge, the above transformation does not induce the gauge transformation because $\theta=\epsilon(v-\beta \cdot A)=0$, and furthermore, thanks to the refined choice of our local time-direction, the fluid looks like entirely at rest. This is the origin of the name hydrostatic. Nevertheless, note that this does not means the system is in a stationary hydrostatic state since we do not assume $\beta^{\mu}$ is a killing vector: $£_{\beta} g_{\mu \nu} \neq 0$. The main reason the hydrostatic gauge gives the most useful gauge is that we can equate the background field in original (real) spacetime with that in (imaginary) thermal spacetime: $\left.e_{\mu}^{a}\right|_{\mathrm{hs}}=\widetilde{e}_{\mu}^{a}$ and $\left.A_{\mu}\right|_{\mathrm{hs}}=\widetilde{A}_{\mu}$. As a result, the above variational formula results in (46) and (47) as

$$
\begin{aligned}
\left\langle\hat{T}_{a}^{\mu}(t, x)\right\rangle_{t}^{\mathrm{LG}} & =\left.\frac{1}{\beta_{0} e} \frac{\delta \Psi[\lambda, j ; t]}{\delta e_{\mu}^{a}(x)}\right|_{\mathrm{hs}}, \\
\left\langle\hat{J}^{\mu}(t, x)\right\rangle_{t}^{\mathrm{LG}} & =\left.\frac{1}{\beta_{0} e} \frac{\delta \Psi[\lambda, j ; t]}{\delta A_{\mu}(x)}\right|_{\mathrm{hs}}-\left.\frac{C}{6} \varepsilon^{\mu v \rho \sigma} A_{\nu} F_{\rho \sigma}\right|_{\mathrm{hs}},
\end{aligned}
$$

which enable us to regard the Massieu-Planck functional as a usual generating functional.

\subsection{Anomaly Matching for Local Thermodynamic Functional}

Based on the obtained formulae, we now discuss the anomaly-induced transport from the point of view of the anomaly matching for the Massieu-Planck functional.

Before moving to the anomaly-induced transport, let us briefly see how we can derive the constitutive relation for a perfect fluid. Employing the simplest power counting scheme $\lambda=O\left(\nabla^{0}\right), j=O\left(\nabla^{0}\right)$, we perform the derivative expansion of the Massieu-Planck function as follows:

$$
\Psi[\lambda, j ; t]=\Psi^{(0)}[\lambda, j ; t]+\Psi^{(1)}[\lambda, j ; t]+O\left(\nabla^{2}\right),
$$


where the superscript represents the number of spatial derivatives acting on parameters $\lambda$ and $j$. Then, the symmetry argument reviewed in the previous subsection tells us that we cannot use the Kaluza-Klein and $U(1)$ gauge fields in the leading-order derivative expansion. As a result, the general form of the leading-order Massieu-Planck functional $\Psi^{(0)}[\lambda, j ; t]$ is expressed as

$$
\Psi^{(0)}[\lambda, j ; t]=\int_{0}^{\beta_{0}} d \tau d^{3} x \widetilde{e} p(\beta, v)=\int d^{3} x \beta^{\prime} \sqrt{\gamma} p(\beta, v),
$$

where $p(\beta, v)$ is a certain function depending on $\beta$ and $v$. By taking the variation with respect to the vierbein and gauge field, we are able to obtain the leading-order constitutive relation as

$$
\left\langle\hat{T}^{\mu v}(t, x)\right\rangle_{(0)}^{\mathrm{LG}}=(e+p) u^{\mu} u^{v}+p g^{\mu v}+O\left(\nabla^{1}\right), \quad\left\langle\hat{J}^{\mu}(t, x)\right\rangle_{(0)}^{\mathrm{LG}}=n u^{\mu}+O\left(\nabla^{1}\right) .
$$

This is nothing but the constitutive relation for the perfect fluid with $e, n, p$ being the energy density, charge density, and fluid pressure, respectively.

Then, the next problem is to specify the first-order derivative correction of the Massieu-Planck functional $\Psi^{(1)}[\lambda, j ; t]$, which is present (absent) in the absence (presence) of the parity symmetry. Since our system is composed of the right-handed Weyl fermion, and thus, there is no parity symmetry, the first-order correction is not prohibited. In this case, two (anomalous) gauge symmetries again plays a central role to extract information on the anomaly-induced transport contained in $\Psi^{(1)}[\lambda, j ; t]$. In the following, after giving a bottom up view relying on the one-loop result in the previous section, we switch to a top down view of the anomaly matching, from which we can derive the anomaly-induced transport beyond the one-loop level.

\subsubsection{Chiral Anomaly in Thermal Spacetime}

At one-loop level, we have already derived the anomaly-induced transport given in Equation (24). On the other hand, we also have the variational formula (47) in a general gauge, or (50) in the hydrostatic gauge. Let us take the hydrostatic gauge. Then, the combination of the above results enables us to obtain the following functional differential equation for $\Psi^{(1)}$ :

$$
\left.\frac{1}{\beta_{0}} \frac{\delta \Psi_{\text {ano }}^{(1)}[\lambda, j ; t]}{\delta A_{i}(x)}\right|_{\mathrm{hs}}+\frac{\mu}{12 \pi^{2}} B^{i}-\frac{1}{12 \pi^{2}} \varepsilon^{0 i j k} A_{k} \partial_{j} \mu=\frac{\mu}{4 \pi^{2}} B^{i}+\left(\frac{\mu^{2}}{4 \pi^{2}}+\frac{T^{2}}{12}\right) \omega^{i},
$$

where we take the flat limit and assume global thermal equilibrium with a constant temperature $\beta_{0}$ in the variational formula. This equation can be easily solved as

$$
\begin{aligned}
\left.\Psi_{\text {ano }}^{(1)}[\lambda, j ; t]\right|_{\mathrm{hs}} ^{\mathrm{eq}} & =\frac{\beta_{0}}{12 \pi^{2}} \int d^{3} x \mu A_{i} B^{i}+\beta_{0} \int d^{3} x\left(\frac{1}{4 \pi^{2}} \mu^{2}+\frac{1}{12} T^{2}\right) A_{i} \omega^{i} \\
& =\frac{\beta_{0}}{12 \pi^{2}} \int d^{3} x \varepsilon^{0 i j k} \mu A_{i} \partial_{j} A_{k}+\frac{\beta_{0}}{2} \int d^{3} x \varepsilon^{0 i j k}\left(\frac{1}{4 \pi^{2}} \mu^{2}+\frac{1}{12} T^{2}\right) A_{i} \partial_{j} u_{k}
\end{aligned}
$$

up to irrelevant constants. On the other hand, we have already clarified that the Massieu-Planck functional need to respect both $U(1)$ and Kaluza-Klein gauge invariance. This constraint then enables us to guess the full result on $\Psi^{(1)}$ for general local thermal equilibrium though Equation (55) is obtained by matching with the one-loop result for linear perturbations on the top of global thermal equilibrium. By using the $U(1)$ and Kaluza-Klein gauge covariant quantities- $\widetilde{A}_{i}^{\prime}$ and $a_{i}$, respectively-together with $\widetilde{A}_{0}=e^{\sigma} \mu$, we specify the first-order derivative correction as

$$
\Psi_{\text {ano }}^{(1)}[\lambda, j ; t]=\frac{C \beta_{0}}{3} \int d^{3} x e \varepsilon^{0 i j k} \widetilde{A}_{0} \widetilde{A}_{i}^{\prime} \partial_{j} \widetilde{A}_{k}^{\prime}+\frac{C \beta_{0}}{6} \int d^{3} x e \varepsilon^{0 i j k} \widetilde{A}_{0}^{2} \widetilde{A}_{i}^{\prime} \partial_{j} a_{k}-\frac{C_{1}}{2 \beta_{0}} \int d^{3} x e \varepsilon^{0 i j k} \widetilde{A}_{i}^{\prime} \partial_{j} a_{k}
$$


with $C_{1} \equiv 1 / 12$. Please note that $\widetilde{A}_{0}$ and $\widetilde{A}_{i}^{\prime}$ defined in Equations (27) and (32) are manifestly Kaluza-Klein gauge invariant quantities.

Let us then confirm the consistency for this result based on the anomaly matching for the Massieu-Planck functional itself. For that purpose, we consider the time-independent gauge transformation given by $\delta_{\theta} \widetilde{A}_{0}=0, \delta_{\theta} \widetilde{A}_{i}=\partial_{i} \theta(x)$. Under this gauge transformation, the Fujikawa method [2] says that the anomalous shift of the Massieu-Planck functional is given by the consistent anomaly:

$$
\delta_{\theta} \Psi[\lambda, j ; t]=-\frac{C \beta_{0}}{3} \int d^{3} x \theta e \varepsilon^{0 i j k} \partial_{i} \widetilde{A}_{0} \partial_{j} \widetilde{A}_{k} .
$$

On the other hand, one can directly show that the first two term of $\Psi_{\text {ano }}^{(1)}[\lambda, j ; t]$ in Equation (56) correctly reproduces this anomalous shift as

$$
\begin{aligned}
\delta_{\theta} \Psi_{\text {ano }}^{(1)}[\lambda, j ; t] & =\frac{C \beta_{0}}{3} \int d^{3} x e \varepsilon^{0 i j k} \widetilde{A}_{0} \partial_{i} \theta \partial_{j} \widetilde{A}_{k}^{\prime}+\frac{C \beta_{0}}{6} \int d^{3} x e \varepsilon^{0 i j k} \widetilde{A}_{0}^{2} \partial_{i} \theta \partial_{j} a_{k} \\
& =-\frac{C \beta_{0}}{3} \int d^{3} x \theta e \varepsilon^{0 i j k} \partial_{i} \widetilde{A}_{0} \partial_{j}\left(\widetilde{A}_{k}-\widetilde{A}_{0} a_{k}\right)-\frac{C \beta_{0}}{3} \int d^{3} x \theta e \varepsilon^{0 i j k} \widetilde{A}_{0} \partial_{i} \widetilde{A}_{0} \partial_{j} a_{k}+\text { (surface terms) } \\
& =-\frac{C \beta_{0}}{3} \int d^{3} x \theta \varepsilon^{0 i j k} \partial_{i} \widetilde{A}_{0} \partial_{j} \widetilde{A}_{k}+(\text { surface terms) }
\end{aligned}
$$

Therefore, we see that the anomalous transport coefficients $C$ proportional to the chemical potential $\mu$ is indeed related to the anomaly coefficient attached to the Weyl fermion.

Nevertheless, the last term in Equation (56), which brings about the CVE proportional to $T^{2}$, is not restricted by the chiral anomaly. From the symmetry point of view, this is just because the last term in Equation (56) remains invariant under the $U(1)$ gauge transformation. This corresponds the fact that the entropy production argument with chiral anomaly leads to the existence of both chiral magnetic and vortical effect [24], in which only the anomalous transport coefficients proportional to the chemical potential are determined. Then, the natural question is "Does the CVE proportional to $T^{2}$ have any relation with the quantum anomaly?"

\subsubsection{Global Anomaly for Kaluza-Klein Gauge Transformation}

It was pointed out the $T^{2}$ term of the chiral vortical coefficient is related to the gravitational contribution to the chiral anomaly [26,56]. However, unlike the chiral magnetic coefficient discussed in this section, it is not clear that how the CVE relates to the $\varepsilon^{\mu v \rho \sigma} R_{\beta \mu \nu}^{\alpha} R_{\alpha \rho \sigma}^{\beta}$, because the number of derivative in $\varepsilon^{\mu \nu \rho \sigma} R_{\beta \mu \nu}^{\alpha} R_{\alpha \rho \sigma}^{\beta}$ is higher than that in $\varepsilon^{\mu v \rho \sigma} F_{\mu \nu} F_{\rho \sigma}$. In other words, $\varepsilon^{\mu v \rho \sigma} R_{\beta \mu \nu}^{\alpha} R_{\alpha \rho \sigma}^{\beta}$ does not directly contribute to the first-order hydrodynamics. An alternative explanation of $T^{2}$ term is that the chiral vortical coefficient is related to a global anomaly $[45,46,106]$. Here, we show the relation between the global anomaly and chiral vortical effect.

As a warm up exercise, let us first consider the global anomaly attached to the Weyl fermion in $1+1$ dimensions, which possesses the chiral anomaly given by

$$
\partial_{\mu} \hat{J}^{\mu}=-\frac{1}{2} C_{2 D} \varepsilon^{\mu v} F_{\mu v} \quad \text { with } \quad C_{2 D} \equiv \frac{1}{2 \pi},
$$

where $\hat{J}^{\mu}$ again denotes the covariant current in $1+1$ dimensional system. In this case, there are no chiral magnetic and vortical effects because there is no transverse direction, and thus, no magnetic field and vorticity. However, there exist nonvanishing $\left\langle\hat{J}^{z}\right\rangle$ and $\left\langle\hat{T}_{z}^{0}\right\rangle$ caused by chiral and global anomalies. The direct calculation at equilibrium shows 


$$
\begin{aligned}
& \left\langle\hat{T}_{z}^{0}\right\rangle_{\mathrm{eq}}=\int_{0}^{\infty} \frac{d p_{z}}{2 \pi} p_{z}\left[n_{F}\left(\left|p_{z}\right|-\mu\right)+n_{F}\left(\left|p_{z}\right|+\mu\right)\right]=\frac{\mu^{2}}{4 \pi}+\frac{\pi}{12} T^{2}, \\
& \left\langle\hat{J}^{z}\right\rangle_{\mathrm{eq}}=\int_{0}^{\infty} \frac{d p_{z}}{2 \pi} \frac{p_{z}}{\left|p_{z}\right|}\left[n_{F}\left(\left|p_{z}\right|-\mu\right)-n_{F}\left(\left|p_{z}\right|+\mu\right)\right]=\frac{\mu}{2 \pi} .
\end{aligned}
$$

On the other hand, the same procedure given above leads to the variational formula in $(1+1)$ dimensions:

$$
\begin{aligned}
\left\langle\hat{T}_{a}^{\mu}\right\rangle_{t}^{\mathrm{LG}} & =\frac{1}{\beta^{\prime} \sqrt{\gamma}} \frac{\delta \Psi[t ; \lambda]}{\delta e_{\mu}^{a}(x)} \\
\left\langle\hat{J}^{\mu}\right\rangle_{t}^{\mathrm{LG}} & =\frac{1}{\beta^{\prime} \sqrt{\gamma}} \frac{\delta \Psi[t ; \lambda]}{\delta \widetilde{A}_{\mu}(x)}-\frac{1}{2} C_{2 D} \widetilde{E}^{\mu v} \widetilde{A}_{v}
\end{aligned}
$$

where $\widetilde{\mathcal{E}}^{\mu v}=N\left(\beta_{0} / \beta^{\prime}\right) \mathcal{E}^{\mu v}$. Then, the matching condition for the momentum density and current results in

$$
\begin{aligned}
& \frac{1}{\beta_{0}} \frac{\delta \Psi_{\text {ano }}}{\delta e_{0}^{z}}=-\frac{1}{\beta_{0}} \frac{\delta \Psi_{\text {ano }}}{\delta a_{z}}=\frac{C_{2 D}}{2} \mu^{2}+\pi C_{1} T^{2}, \\
& \frac{1}{\beta_{0}} \frac{\delta \Psi_{\text {ano }}}{\delta A_{z}}+\frac{C_{2 D}}{2} \mu=C_{2 D} \mu .
\end{aligned}
$$

Solving Equations (62) and (63), we find

$$
\Psi_{\text {ano }}=\frac{C_{2 D} \beta_{0}}{2} \int d z \widetilde{A}_{0} \widetilde{A}_{z}^{\prime}-\pi \frac{C_{1}}{\beta_{0}} \int d z a_{z} .
$$

This gives the anomalous part of the Masseiu-Planck functional. To detect anomalies, we compactify the spatial direction with the length $L$. Here we will show $\Psi_{\text {ano }}$ has two types of anomalies. One is the chiral anomaly: Under $U(1)$ gauge transformation $\widetilde{A}_{z} \rightarrow \widetilde{A}_{z}+\partial_{z} \theta(z)$, the anomalous shift of $\Psi$ arises:

$$
\delta_{\theta} \Psi_{\text {ano }}=-\frac{C_{2 D} \beta_{0}}{2} \int d z \theta \partial_{z} \widetilde{A}_{0}
$$

which correctly reproduces the consistent anomaly in thermal spacetime. The other is the global anomaly associated with the Kaluza-Klein gauge transformation:

$$
\left\{\begin{array}{l}
\widetilde{t} \rightarrow \widetilde{t}+\chi(z), \\
a_{z} \rightarrow a_{z}-\partial_{z} \chi(z),
\end{array}\right.
$$

where $\widetilde{A}_{z}^{\prime}$ remains invariant. Under this transformation, $\Psi_{\text {ano }}$ also acquires the anomalous shift given by

$$
\delta_{\chi} \Psi_{\text {ano }}=\pi \frac{C_{1}}{\beta_{0}} \int d z \partial_{z} \chi(z)
$$

which is just a boundary term, so that $\Psi_{\text {ano }}$ is invariant under local transformation with $\chi(0)=$ $\chi(L)$. However, if we consider global transformation, $\chi(z)=-2 i \beta_{0} z / L$, which corresponds to the imaginary-time shift $\tau \rightarrow \tau+2 z \beta_{0} / L$ that keep the boundary condition, we have an additional phase

$$
\Psi_{\mathrm{ano}} \rightarrow \Psi_{\mathrm{ano}}-2 \pi i C_{1}
$$


which can be understood as the global anomaly associated with the large diffeomorphism. This anomalous phase is related to the three-dimensional gravitational Chern-Simons term through the anomaly inflow mechanism, which is also related to the gravitational contribution to chiral anomaly in $3+1$ dimensions $[107,108]$.

This argument can be generalized to higher dimensions. In $(3+1)$ dimensions, $\Psi_{\text {ano }}$ is given in Equation (56). To detect the global anomaly, we compactify the space to $S^{1} \times S^{2}$, where we choose $z$ as the coordinate on $S^{1}$. Under the large diffeomorphism, $\tau \rightarrow \tau+2 z \beta_{0} / L$, the term contributing to the $T^{2}$ part of CVE transforms as

$$
\Psi_{\text {ano }} \rightarrow \Psi_{\text {ano }}-2 \pi i C_{1} \int \frac{d^{2} x}{2 \pi} e \varepsilon^{0 i j z} \partial_{i} \widetilde{A}_{j}^{\prime} .
$$

This is the global mixed anomaly between $U(1)$ gauge and large diffeomorphism. Therefore, we see that the chiral vortical coefficient proportional to $T^{2}$, which is nothing but $C_{1}$, is related to the mixed global anomaly. Nevertheless, it should be noted that the mixed global anomaly only fixes a "fractional" part of $T^{2}$ term. This is because a shift $C_{1} \rightarrow C_{1}+n$ with $n \in \mathbb{Z}$ does not change the partition functional [54].

\section{Summary and Discussion}

In this paper, we have discussed two approaches to derive the anomaly-induced transport phenomena for the system composed of a Weyl fermion: perturbative evaluation of the chiral magnetic/vortical conductivity with the help of the (equilibrium) linear response theory, and the nonperturbative determination of anomalous parts of the local thermodynamic functional on the basis of the anomaly matching. Both derivations are based on the imaginary-time formalism of the quantum field theory, and we have seen that the obtained anomalous constitutive relations correctly describe the chiral magnetic/vortical effect. Although it is not so clear in the first derivation, the second derivation shows that the chiral magnetic/vortical effect results from the first-order derivative corrections of the local thermodynamic functional, and thus, they are clearly nondissipative in nature. This is perfectly consistent with the known result obtained from the hydrostatic partition function method [29-32,35,38-40,43,45,46], and we rigorously clarify why that method works well. This local equilibrium part of the constitutive relation also complete the application of Zubarev's nonequilibrium statistical operator method to derive the hydrodynamic equation for the parity-violating (anomalous) fluid.

There are several interesting questions related to the current work. It has been already pointed out that the coefficient in front of the $T^{2}$-term of the CVE will be renormalized in the presence of dynamical gauge fields such as the gluon in the QCD plasma [109]. It may be interesting to examine which part of the anomaly matching argument associated with the large diffeomorphism (Kaluza-Klein gauge) transformation should be modified due to the existence of the dynamical gauge field. Another important issue associated with the inclusion of dynamical electromagnetic field is its dynamics. When we consider the dynamics of the electromagnetic field rather than treating it as the background one, we encounter with several interesting phenomena such as the chiral plasma instability [110-114], and mixing of some hydrodynamic modes (chiral magnetic wave) to be the massive collective excitation (chiral plasmon) $[48,65,115,116]$. It is desirable to systematically describe them based on the generalization of magnetohydrodynamics for the chiral plasma by formulating chiral magnetohydrodynamics. Chiral magnetohydrodynamics is just recently formulated based on e.g., the phenomenological entropy-current analysis [117] (See also Refs. [118-124]), but less is clarified from the underlying quantum field theory. Combined with the recent development of the magnetohydrodynamics itself from the field theoretical viewpoint [125-130], it may be interesting to formulate chiral magnetohydrodynamics based on the Zubarev's nonequilibrium statistical operator method equipped with the path-integral formula for the local thermodynamic functional reviewed in this paper. 
Author Contributions: All authors have substantially contributed to the research reported in this work and the writing of the manuscript.

Funding: This research was funded by Japan Society of Promotion of Science (JSPS) Grant-in-Aid for Scientific Research (KAKENHI) Grant Numbers JP16K17716, 17H06462, 18H01211, and 18H01217, and also supported by the Ministry of Education, Culture, Sports, Science, and Technology(MEXT)-Supported Program for the Strategic Research Foundation at Private Universities "Topological Science" (Grant No. S1511006).

Acknowledgments: M.H. was supported by the Special Postdoctoral Researchers Program at RIKEN. This work was partially supported by the RIKEN iTHES/iTHEMS Program, in particular, iTHEMS STAMP working group.

Conflicts of Interest: The authors declare no conflict of interest.

\section{References}

1. Bertlmann, R.A. Anomalies in Quantum Field Theory; Oxford University Press: Oxford, UK, 2000; Volume 91.

2. Fujikawa, K.; Suzuki, H. Path Integrals and Quantum Anomalies; Oxford University Press on Demand: Oxford, UK, 2004; Volume 122.

3. Fukuda, H.; Miyamoto, Y. On the $\gamma$-Decay of Neutral Meson. Prog. Theor. Phys. 1949, 4, 347-357. [CrossRef]

4. Adler, S.L. Axial vector vertex in spinor electrodynamics. Phys. Rev. 1969, 177, 2426-2438. [CrossRef]

5. Bell, J.S.; Jackiw, R. A PCAC puzzle: $\pi^{0} \rightarrow \gamma \gamma$ in the $\sigma$ model. Nuovo Cim. 1969, A60, 47-61. [CrossRef]

6. 't Hooft, G. Naturalness, chiral symmetry, and spontaneous chiral symmetry breaking. In Recent Developments in Gauge Theories. NATO Advanced Study Institutes Series (Series B. Physics); Springer: Boston, MA, USA, 1979; Volume 59. [CrossRef]

7. Frishman, Y.; Schwimmer, A.; Banks, T.; Yankielowicz, S. The Axial Anomaly and the Bound State Spectrum in Confining Theories. Nucl. Phys. 1981, B177, 157-171. [CrossRef]

8. Wen, X.G. Classifying gauge anomalies through symmetry-protected trivial orders and classifying gravitational anomalies through topological orders. Phys. Rev. 2013, D88, 045013. [CrossRef]

9. Tachikawa, Y.; Yonekura, K. On time-reversal anomaly of 2+1d topological phases. PTEP 2017, 2017,033 B04. [CrossRef]

10. Gaiotto, D.; Kapustin, A.; Komargodski, Z.; Seiberg, N. Theta, Time Reversal, and Temperature. J. High Energy Phys. 2017, 5, 091. [CrossRef]

11. Tanizaki, Y.; Kikuchi, Y. Vacuum structure of bifundamental gauge theories at finite topological angles. J. High Energy Phys. 2017, 6, 102. [CrossRef]

12. Shimizu, H.; Yonekura, K. Anomaly constraints on deconfinement and chiral phase transition. Phys. Rev. 2018, D97, 105011. [CrossRef]

13. Tanizaki, Y.; Misumi, T.; Sakai, N. Circle compactification and 't Hooft anomaly. J. High Energy Phys. 2017, 12, 56. [CrossRef]

14. Tanizaki, Y.; Kikuchi, Y.; Misumi, T.; Sakai, N. Anomaly matching for phase diagram of massless $\mathbb{Z}_{N}-Q C D$. Phys. Rev. 2018, D97, 054012. [CrossRef]

15. Sulejmanpasic, T.; Tanizaki, Y. C-P-T anomaly matching in bosonic quantum field theory and spin chains. Phys. Rev. 2018, B97, 144201. [CrossRef]

16. Yao, Y.; Hsieh, C.T.; Oshikawa, M. Anomaly matching and symmetry-protected critical phases in $S U(N)$ spin systems in $1+1$ dimensions. arXiv 2018, arXiv:1805.06885.

17. Tanizaki, Y.; Sulejmanpasic, T. Anomaly and global inconsistency matching: $\theta$-angles, $S U(3) / U(1)^{2}$ nonlinear sigma model, SU(3) chains and its generalizations. Phys. Rev. 2018, B98, 115126. [CrossRef]

18. Tanizaki, Y. Anomaly constraint on massless QCD and the role of Skyrmions in chiral symmetry breaking. J. High Energy Phys. 2018, 8, 171. [CrossRef]

19. Yonekura, K. Anomaly matching in QCD thermal phase transition. arXiv 2019, arXiv:1901.08188.

20. Fukushima, K.; Kharzeev, D.E.; Warringa, H.J. The Chiral Magnetic Effect. Phys. Rev. 2008, D78, 074033. [CrossRef]

21. Erdmenger, J.; Haack, M.; Kaminski, M.; Yarom, A. Fluid dynamics of R-charged black holes. J. High Energy Phys. 2009, 1, 55. [CrossRef]

22. Banerjee, N.; Bhattacharya, J.; Bhattacharyya, S.; Dutta, S.; Loganayagam, R.; Surowka, P. Hydrodynamics from charged black branes. J. High Energy Phys. 2011, 1, 094. [CrossRef] 
23. Torabian, M.; Yee, H.U. Holographic nonlinear hydrodynamics from AdS/CFT with multiple/non-Abelian symmetries. J. High Energy Phys. 2009, 8, 20. [CrossRef]

24. Son, D.T.; Surowka, P. Hydrodynamics with Triangle Anomalies. Phys. Rev. Lett. 2009, $103,191601$. [CrossRef] [PubMed]

25. Amado, I.; Landsteiner, K.; Pena-Benitez, F. Anomalous transport coefficients from Kubo formulas in Holography. J. High Energy Phys. 2011, 5, 081. [CrossRef]

26. Landsteiner, K.; Megias, E.; Pena-Benitez, F. Gravitational Anomaly and Transport. Phys. Rev. Lett. 2011, 107, 021601. [CrossRef]

27. Gao, J.H.; Liang, Z.T.; Pu, S.; Wang, Q.; Wang, X.N. Chiral Anomaly and Local Polarization Effect from Quantum Kinetic Approach. Phys. Rev. Lett. 2012, 109, 232301. [CrossRef]

28. Son, D.T.; Yamamoto, N. Berry Curvature, Triangle Anomalies, and the Chiral Magnetic Effect in Fermi Liquids. Phys. Rev. Lett. 2012, 109, 181602. [CrossRef]

29. Banerjee, N.; Bhattacharya, J.; Bhattacharyya, S.; Jain, S.; Minwalla, S.; Sharma, T. Constraints on Fluid Dynamics from Equilibrium Partition Functions. J. High Energy Phys. 2012, 9, 46. [CrossRef]

30. Jensen, K.; Kaminski, M.; Kovtun, P.; Meyer, R.; Ritz, A.; Yarom, A. Towards hydrodynamics without an entropy current. Phys. Rev. Lett. 2012, 109, 101601. [CrossRef]

31. Jensen, K. Triangle Anomalies, Thermodynamics, and Hydrodynamics. Phys. Rev. 2012, D85, 125017. [CrossRef]

32. Banerjee, N.; Dutta, S.; Jain, S.; Loganayagam, R.; Sharma, T. Constraints on Anomalous Fluid in Arbitrary Dimensions. J. High Energy Phys. 2013, 3, 48. [CrossRef]

33. Stephanov, M.A.; Yin, Y. Chiral Kinetic Theory. Phys. Rev. Lett. 2012, 109, 162001. [CrossRef] [PubMed]

34. Landsteiner, K.; Megias, E.; Pena-Benitez, F. Anomalous Transport from Kubo Formulae. Lect. Notes Phys. 2013, 871, 433-468. [CrossRef]

35. Jensen, K.; Loganayagam, R.; Yarom, A. Thermodynamics, gravitational anomalies and cones. J. High Energy Phys. 2013, 2, 88. [CrossRef]

36. Son, D.T.; Yamamoto, N. Kinetic theory with Berry curvature from quantum field theories. Phys. Rev. 2013, D87, 085016. [CrossRef]

37. Chen, J.W.; Pu, S.; Wang, Q.; Wang, X.N. Berry Curvature and Four-Dimensional Monopoles in the Relativistic Chiral Kinetic Equation. Phys. Rev. Lett. 2013, 110, 262301. [CrossRef]

38. Jensen, K.; Kovtun, P.; Ritz, A. Chiral conductivities and effective field theory. J. High Energy Phys. 2013, 10, 186. [CrossRef]

39. Jensen, K.; Loganayagam, R.; Yarom, A. Anomaly inflow and thermal equilibrium. J. High Energy Phys. 2014, 5, 134. [CrossRef]

40. Jensen, K.; Loganayagam, R.; Yarom, A. Chern-Simons terms from thermal circles and anomalies. J. High Energy Phys. 2014, 5, 110. [CrossRef]

41. Manuel, C.; Torres-Rincon, J.M. Kinetic theory of chiral relativistic plasmas and energy density of their gauge collective excitations. Phys. Rev. 2014, D89, 096002. [CrossRef]

42. Chen, J.Y.; Son, D.T.; Stephanov, M.A.; Yee, H.U.; Yin, Y. Lorentz Invariance in Chiral Kinetic Theory. Phys. Rev. Lett. 2014, 113, 182302. [CrossRef] [PubMed]

43. Haehl, F.M.; Loganayagam, R.; Rangamani, M. Adiabatic hydrodynamics: The eightfold way to dissipation. J. High Energy Phys. 2015, 5, 060. [CrossRef]

44. Chen, J.Y.; Son, D.T.; Stephanov, M.A. Collisions in Chiral Kinetic Theory. Phys. Rev. Lett. 2015, 115, 021601. [CrossRef] [PubMed]

45. Golkar, S.; Sethi, S. Global Anomalies and Effective Field Theory. J. High Energy Phys. 2016, 5, 105. [CrossRef]

46. Chowdhury, S.D.; David, J.R. Global gravitational anomalies and transport. J. High Energy Phys. 2016, 12, 116. [CrossRef]

47. Landsteiner, K. Notes on Anomaly Induced Transport. Acta Phys. Polon. 2016, B47, 2617. [CrossRef]

48. Gorbar, E.V.; Miransky, V.A.; Shovkovy, I.A.; Sukhachov, P.O. Consistent Chiral Kinetic Theory in Weyl Materials: Chiral Magnetic Plasmons. Phys. Rev. Lett. 2017, 118, 127601. [CrossRef] [PubMed]

49. Hidaka, Y.; Pu, S.; Yang, D.L. Relativistic Chiral Kinetic Theory from Quantum Field Theories. Phys. Rev. 2017, D95, 091901. [CrossRef]

50. Buzzegoli, M.; Grossi, E.; Becattini, F. General equilibrium second-order hydrodynamic coefficients for free quantum fields. J. High Energy Phys. 2017, 10, 091. [CrossRef] 
51. Hidaka, Y.; Pu, S.; Yang, D.L. Nonlinear Responses of Chiral Fluids from Kinetic Theory. Phys. Rev. 2018, D97, 016004. [CrossRef]

52. Mueller, N.; Venugopalan, R. The chiral anomaly, Berry's phase and chiral kinetic theory, from world-lines in quantum field theory. Phys. Rev. 2018, D97, 051901. [CrossRef]

53. Mueller, N.; Venugopalan, R. Worldline construction of a covariant chiral kinetic theory. Phys. Rev. 2017, D96, 016023. [CrossRef]

54. Glorioso, P.; Liu, H.; Rajagopal, S. Global Anomalies, Discrete Symmetries, and Hydrodynamic Effective Actions. J. High Energy Phys. 2019, 1, 043. [CrossRef]

55. Hidaka, Y.; Yang, D.L. Nonequilibrium chiral magnetic/vortical effects in viscous fluids. Phys. Rev. 2018, D98, 016012. [CrossRef]

56. Stone, M.; Kim, J. Mixed Anomalies: Chiral Vortical Effect and the Sommerfeld Expansion. Phys. Rev. 2018, D98, 025012. [CrossRef]

57. Carignano, S.; Manuel, C.; Torres-Rincon, J.M. Consistent relativistic chiral kinetic theory: A derivation from on-shell effective field theory. Phys. Rev. 2018, D98, 076005. [CrossRef]

58. Buzzegoli, M.; Becattini, F. General thermodynamic equilibrium with axial chemical potential for the free Dirac field. J. High Energy Phys. 2018, 12, 002. [CrossRef]

59. Dayi, O.F.; Kilinçarslan, E. Quantum Kinetic Equation in the Rotating Frame and Chiral Kinetic Theory. Phys. Rev. 2018, D98, 081701. [CrossRef]

60. Liu, Y.C.; Gao, L.L.; Mameda, K.; Huang, X.G. Chiral kinetic theory in curved spacetime. arXiv 2018, arXiv:1812.10127.

61. Mueller, N.; Venugopalan, R. Constructing phase space distributions with internal symmetries. arXiv 2019, arXiv:1901.10492.

62. Vilenkin, A. Macroscopic parity violating effects: Neutrino fluxes from rotating black holes and in rotating thermal radiation. Phys. Rev. 1979, D20, 1807-1812. [CrossRef]

63. Vilenkin, A. Equilibrium parity violating current in a magnetic field. Phys. Rev. 1980, D22, 3080-3084. [CrossRef]

64. Kharzeev, D.E.; McLerran, L.D.; Warringa, H.J. The Effects of topological charge change in heavy ion collisions: 'Event by event P and CP violation'. Nuclear Phys. 2008, A803, 227-253. [CrossRef]

65. Kharzeev, D.E.; Yee, H.U. Chiral Magnetic Wave. Phys. Rev. 2011, D83, 085007. [CrossRef]

66. Burnier, Y.; Kharzeev, D.E.; Liao, J.; Yee, H.U. Chiral magnetic wave at finite baryon density and the electric quadrupole moment of quark-gluon plasma in heavy ion collisions. Phys. Rev. Lett. 2011, 107, 052303. [CrossRef]

67. Hongo, M.; Hirono, Y.; Hirano, T. Anomalous-hydrodynamic analysis of charge-dependent elliptic flow in heavy-ion collisions. Phys. Lett. 2017, B775, 266-270. [CrossRef]

68. Yee, H.U.; Yin, Y. Realistic Implementation of Chiral Magnetic Wave in Heavy Ion Collisions. Phys. Rev. 2014, C89, 044909. [CrossRef]

69. Hirono, Y.; Hirano, T.; Kharzeev, D.E. The chiral magnetic effect in heavy-ion collisions from event-by-event anomalous hydrodynamics. arXiv 2014, arXiv:1412.0311.

70. Adamczyk, L.; Adkins, J.K.; Agakishiev, G.; Aggarwal, M.M. Observation of charge asymmetry dependence of pion elliptic flow and the possible chiral magnetic wave in heavy-ion collisions. Phys. Rev. Lett. 2015, 114, 252302. [CrossRef] [PubMed]

71. Yin, Y.; Liao, J. Hydrodynamics with chiral anomaly and charge separation in relativistic heavy ion collisions. Phys. Lett. 2016, B756, 42-46. [CrossRef]

72. Huang, X.G. Electromagnetic fields and anomalous transports in heavy-ion collisions-A pedagogical review. Rept. Prog. Phys. 2016, 79, 076302. [CrossRef]

73. Kharzeev, D.E.; Liao, J.; Voloshin, S.A.; Wang, G. Chiral magnetic and vortical effects in high-energy nuclear collisions-A status report. Prog. Part. Nuclear Phys. 2016, 88, 1-28. [CrossRef]

74. Shi, S.; Jiang, Y.; Lilleskov, E.; Liao, J. Anomalous Chiral Transport in Heavy Ion Collisions from Anomalous-Viscous Fluid Dynamics. Ann. Phys. 2018, 394, 50-72. [CrossRef]

75. Charbonneau, J.; Zhitnitsky, A. Topological Currents in Neutron Stars: Kicks, Precession, Toroidal Fields, and Magnetic Helicity. J. Cosmol. Astropart. Phys. 2010, 1008, 010. [CrossRef]

76. Grabowska, D.; Kaplan, D.B.; Reddy, S. Role of the electron mass in damping chiral plasma instability in Supernovae and neutron stars. Phys. Rev. 2015, D91, 085035. [CrossRef] 
77. Kaminski, M.; Uhlemann, C.F.; Bleicher, M.; Schaffner-Bielich, J. Anomalous hydrodynamics kicks neutron stars. Phys. Lett. 2016, B760, 170-174. [CrossRef]

78. Sigl, G.; Leite, N. Chiral Magnetic Effect in Protoneutron Stars and Magnetic Field Spectral Evolution. J. Cosmol. Astropart. Phys. 2016, 1601, 025. [CrossRef]

79. Yamamoto, N. Chiral transport of neutrinos in supernovae: Neutrino-induced fluid helicity and helical plasma instability. Phys. Rev. 2016, D93, 065017. [CrossRef]

80. Masada, Y.; Kotake, K.; Takiwaki, T.; Yamamoto, N. Chiral magnetohydrodynamic turbulence in core-collapse supernovae. Phys. Rev. 2018, D98, 083018. [CrossRef]

81. Zyuzin, A.A.; Burkov, A.A. Topological response in Weyl semimetals and the chiral anomaly. Phys. Rev. 2012, B86, 115133. [CrossRef]

82. Goswami, P.; Tewari, S. Axionic field theory of (3+1)-dimensional Weyl semimetals. Phys. Rev. 2013, B88, 245107. [CrossRef]

83. Chen, Y.; Wu, S.; Burkov, A.A. Axion response in Weyl semimetals. Phys. Rev. 2013, B88, 125105. [CrossRef]

84. Basar, G.; Kharzeev, D.E.; Yee, H.U. Triangle anomaly in Weyl semimetals. Phys. Rev. 2014, B89, 035142. [CrossRef]

85. Hosur, P.; Qi, X. Recent developments in transport phenomena in Weyl semimetals. Comptes Rendus Phys. 2013, 14, 857-870. [CrossRef]

86. Landsteiner, K. Anomalous transport of Weyl fermions in Weyl semimetals. Phys. Rev. 2014, B89, 075124. [CrossRef]

87. Chernodub, M.N.; Cortijo, A.; Grushin, A.G.; Landsteiner, K.; Vozmediano, M.A.H. Condensed matter realization of the axial magnetic effect. Phys. Rev. 2014, B89, 081407. [CrossRef]

88. Gorbar, E.V.; Miransky, V.A.; Shovkovy, I.A. Chiral anomaly, dimensional reduction, and magnetoresistivity of Weyl and Dirac semimetals. Phys. Rev. 2014, B89, 085126. [CrossRef]

89. Armitage, N.P.; Mele, E.J.; Vishwanath, A. Weyl and Dirac Semimetals in Three Dimensional Solids. Rev. Mod. Phys. 2018, 90, 015001. [CrossRef]

90. Gorbar, E.V.; Miransky, V.A.; Shovkovy, I.A.; Sukhachov, P.O. Anomalous transport properties of Dirac and Weyl semimetals (Review Article). Low Temp. Phys. 2018, 44, 487-505. [CrossRef]

91. Li, Q.; Kharzeev, D.E.; Zhang, C.; Huang, Y.; Pletikosic, I.; Fedorov, A.V.; Zhong, R.D.; Schneeloch, J.A.; Gu, G.D.; Valla, T. Observation of the chiral magnetic effect in ZrTe5. Nat. Phys. 2016, 12, 550-554. [CrossRef]

92. Lv, B.Q.; Weng, H.M.; Fu, B.B.; Wang, X.P.; Miao, H.; Ma, J. Experimental discovery of Weyl semimetal TaAs. Phys. Rev. 2015, X5, 031013. [CrossRef]

93. Xu, S.Y.; Belopolski, I.; Alidoust, N.; Neupane, M.; Bian, G.; Zhang, C.; Sank, R. Discovery of a Weyl Fermion semimetal and topological Fermi arcs. Science 2015, 349, 613-617. [CrossRef]

94. Matsubara, T. A New Approach to Quantum-Statistical Mechanics. Prog. Theor. Phys. 1955, 14, $351-378$. [CrossRef]

95. Abrikosov, A.A.; Gorkov, L.P.; Dzyaloshinskii, I.E. On the Application of Quantum-Field-Theory Methods to Problems of Quantum Statistics at Finite Temperatures. Sov. Phys. JETP 1959, 9, 636-641.

96. Le Bellac, M. Thermal Field Theory; Cambridge University Press: Cambridge, UK, 2000.

97. Kapusta, J.I.; Gale, C. Finite-Temperature Field Theory: Principles and Applications; Cambridge University Press: Cambridge, UK, 2006.

98. Zubarev, D.N.; Prozorkevich, A.V.; Smolyanskii, S.A. Derivation of nonlinear generalized equations of quantum relativistic hydrodynamics. Theor. Math. Phys. 1979, 40, 821-831. [CrossRef]

99. Zubarev, D.N.; Morozov, V.; Ropke, G. Statistical Mechanics of Nonequilibrium Processes, Volume 1: Basic Concepts, Kinetic Theory, 1st ed.; Wiley-VCH: Hoboken, NJ, USA, 1996.

100. Zubarev, D.N.; Morozov, V.; Ropke, G. Statistical Mechanics of Nonequilibrium Processes, Volume 2: Relaxation and Hydrodynamic Processes; Wiley-VCH: Hoboken, NJ, USA, 1997.

101. Becattini, F.; Bucciantini, L.; Grossi, E.; Tinti, L. Local thermodynamical equilibrium and the beta frame for a quantum relativistic fluid. Eur. Phys. J. 2015, C75, 191. [CrossRef]

102. Hayata, T.; Hidaka, Y.; Noumi, T.; Hongo, M. Relativistic hydrodynamics from quantum field theory on the basis of the generalized Gibbs ensemble method. Phys. Rev. 2015, D92, 065008. [CrossRef]

103. Hongo, M. Path-integral formula for local thermal equilibrium. Ann. Phys. 2017, 383, 1-32. [CrossRef]

104. Hongo, M. Nonrelativistic Hydrodynamics from Quantum Field Theory: (I) Normal Fluid Composed of Spinless Schrödinger Fields. J. Stat. Phys. 2019. [CrossRef] 
105. Bardeen, W.A.; Zumino, B. Consistent and Covariant Anomalies in Gauge and Gravitational Theories. Nucl. Phys. 1984, B244, 421-453. [CrossRef]

106. Nakai, R.; Ryu, S.; Nomura, K. Laughlin's argument for the quantized thermal Hall effect. Phys. Rev. 2017, B95, 165405. [CrossRef]

107. Witten, E. Global Aspects of Current Algebra. Nucl. Phys. 1983, B223, 422-432. [CrossRef]

108. Witten, E. Global gravitational anomalies. Commun. Math. Phys. 1985, 100, 197. [CrossRef]

109. Golkar, S.; Son, D.T. (Non)-renormalization of the chiral vortical effect coefficient. J. High Energy Phys. 2015, 2, 169. [CrossRef]

110. Boyarsky, A.; Frohlich, J.; Ruchayskiy, O. Self-consistent evolution of magnetic fields and chiral asymmetry in the early Universe. Phys. Rev. Lett. 2012, 108, 031301. [CrossRef]

111. Tashiro, H.; Vachaspati, T.; Vilenkin, A. Chiral Effects and Cosmic Magnetic Fields. Phys. Rev. 2012, D86, 105033. [CrossRef]

112. Akamatsu, Y.; Yamamoto, N. Chiral Plasma Instabilities. Phys. Rev. Lett. 2013, 111, 052002. [CrossRef]

113. Akamatsu, Y.; Yamamoto, N. Chiral Langevin theory for non-Abelian plasmas. Phys. Rev. 2014, D90, 125031. [CrossRef]

114. Manuel, C.; Torres-Rincon, J.M. Dynamical evolution of the chiral magnetic effect: Applications to the quark-gluon plasma. Phys. Rev. 2015, D92, 074018. [CrossRef]

115. Gorbar, E.V.; Miransky, V.A.; Shovkovy, I.A.; Sukhachov, P.O. Chiral magnetic plasmons in anomalous relativistic matter. Phys. Rev. 2017, B95, 115202. [CrossRef]

116. Rybalka, D.; Gorbar, E.; Shovkovy, I. Hydrodynamic modes in a magnetized chiral plasma with vorticity. Phys. Rev. 2019, D99, 016017. [CrossRef]

117. Hattori, K.; Hirono, Y.; Yee, H.U.; Yin, Y. MagnetoHydrodynamics with chiral anomaly: Phases of collective excitations and instabilities. arXiv 2017, arXiv:1711.08450.

118. Giovannini, M.; Shaposhnikov, M.E. Primordial hypermagnetic fields and triangle anomaly. Phys. Rev. 1998 D57, 2186. [CrossRef]

119. Giovannini, M. Anomalous Magnetohydrodynamics. Phys. Rev. 2013, D88, 063536. [CrossRef]

120. Boyarsky, A.; Frohlich, J.; Ruchayskiy, O. Magnetohydrodynamics of Chiral Relativistic Fluids. Phys. Rev. 2015, D92, 043004. [CrossRef]

121. Gorbar, E.V.; Shovkovy, I.A.; Vilchinskii, S.; Rudenok, I.; Boyarsky, A.; Ruchayskiy, O. Anomalous Maxwell equations for inhomogeneous chiral plasma. Phys. Rev. 2016, D93, 105028. [CrossRef]

122. Yamamoto, N. Scaling laws in chiral hydrodynamic turbulence. Phys. Rev. 2016, D93, 125016. [CrossRef]

123. Giovannini, M. Anomalous magnetohydrodynamics in the extreme relativistic domain. Phys. Rev. 2016, D94, 081301. [CrossRef]

124. Rogachevskii, I.; Ruchayskiy, O.; Boyarsky, A.; Fröhlich, J.; Kleeorin, N.; Brandenburg, A.; Schober, J. Laminar and turbulent dynamos in chiral magnetohydrodynamics-I: Theory. Astrophys. J. 2017, 846, 153. [CrossRef]

125. Huang, X.G.; Sedrakian, A.; Rischke, D.H. Kubo formulae for relativistic fluids in strong magnetic fields. Ann. Phys. 2011, 326, 3075-3094. [CrossRef]

126. Grozdanov, S.; Hofman, D.M.; Iqbal, N. Generalized global symmetries and dissipative magnetohydrodynamics. Phys. Rev. 2017, D95, 096003. [CrossRef]

127. Hernandez, J.; Kovtun, P. Relativistic magnetohydrodynamics. J. High Energy Phys. 2017, 5, 001. [CrossRef]

128. Armas, J.; Jain, A. Magnetohydrodynamics as superfluidity. arXiv 2018, arXiv:1808.01939.

129. Glorioso, P.; Son, D.T. Effective field theory of magnetohydrodynamics from generalized global symmetries. arXiv 2018, arXiv:1811.04879.

130. Armas, J.; Jain, A. One-form superfluids \& magnetohydrodynamics. arXiv 2018, arXiv:1811.04913.

(C) 2019 by the authors. Licensee MDPI, Basel, Switzerland. This article is an open access article distributed under the terms and conditions of the Creative Commons Attribution (CC BY) license (http:/ / creativecommons.org/licenses/by/4.0/). 